\title{
SHOCK CAPTURING VISCOSITIES FOR THE GENERAL FLUID MECHANICS ALGORITHM
}

\author{
P. NITHIARASU ${ }^{a}$, O.C. ZIENKIEWICZ ${ }^{\mathrm{a}, *}$, B.V.K. SATYA SAI ${ }^{\mathrm{a}}$, K. MORGAN ${ }^{\mathrm{a}}$, \\ R. CODINA ${ }^{\mathrm{b}}$ AND M. VÁZQUEZ ${ }^{\mathrm{b}}$ \\ ${ }^{a}$ Institute for Numerical Methods in Engineering, University of Wales Swansea, Swansea SA2 8PP, UK \\ ${ }^{\mathrm{b}}$ International Center for Numerical Methods in Engineering, Universidad Politécnica de Catalunya, Barcelona, Spain
}

\section{SUMMARY}

The performance of different shock capturing viscosities has been examined using our general fluid mechanics algorithm. Four different schemes have been tested, both for viscous and inviscid compressible flow problems. Results show that the methods based on the second gradient of pressure give better performance in all situations. For instance, the method constructed from the nodal pressure values and consistent and lumped mass matrices is an excellent choice for inviscid problems. The method based on $L_{2}$ projection is better than any other method in viscous flow computations. The residual based anisotropic method gives excellent performance in the supersonic range and gives better results in the hypersonic regime if a small amount of residual smoothing is used. C) 1998 John Wiley \& Sons, Ltd.

KEY WORDS: finite elements; compressible flow; artificial viscosity

\section{INTRODUCTION}

Second-order schemes for high-speed compressible flow problems with error in the solution of the order of $O\left(h^{2}\right)$ lead to unavoidable high frequency oscillations in the vicinity of shocks. The first-order schemes, on the other hand, with the error magnitude $O(h)$, damp the high-frequency components of solutions. To overcome this deficiency of higher order schemes, many shock capturing schemes are introduced in literature. The excellent summary of shock capturing viscosity methods for finite difference based methods has been given by Hirsch [1]. Woodward and Colella [2] compared the performance of three different techniques namely those of artificial viscosity, linear hybridization and Godunov's approach. Among the three, the artificial viscosity method is the most direct procedure for shock capturing. Recent developments of high resolution methods such as TVD [3] and MUSCL [4] schemes in finite element computations are observed to be good in supersonic as well as in hypersonic flow ranges [5]. However, a disadvantage of these schemes is that higher computational time is required compared with standard artificial viscosity schemes.

The shock capturing viscosity scheme was first introduced by Von Neumann and Richtmyer [6]. This method consists of representing the discontinuity as a narrow region of steep gradients in the flow variables. Stabilization is achieved by adding a suitable artificial dissipation term

* Correspondence to: Department of Civil Engineering, University of Wales Swansea, Singleton Park, Swansea SA2 $8 \mathrm{PP}, \mathrm{UK}$.

CCC 0271-2091/98/191325-29\$17.50

Received February 1998

(C) 1998 John Wiley \& Sons, Ltd.

Revised February 1998 
that mimics the action of viscosity in the neighbourhood of shocks. Other significant developments in this area are those of Lapidus [7], Steger [8], MacCormack and Baldwin [9] and Jameson [10]. In the finite difference literature, artificial viscosity terms are generalised by a coefficient having the dimension of velocity and multiplied by $h$ (element size) to get the dimension of viscosity $\left(\mu_{a}\right)$ At Swansea, a modified form of the method based on second gradient of pressure has been developed by Peraire et al. [11] and Morgan et al. [12] for finite element computations. This modified form of viscosity with the pressure switch calculated from the nodal pressure values is used subsequently in compressible flow calculations $[5,13,14]$. Recently, anisotropic shock capturing has been introduced $[15,16]$ to add diffusion in a more rational way. Recent results using this approach [17] are encouraging and further investigation of different inviscid and viscous problems is essential. In this article, a search for good artificial viscosity schemes is undertaken for the general fluid mechanics algorithm introduced by Zienkiewicz and Codina [16] and the performance of different artificial viscosity schemes is investigated.

\section{GOVERNING EQUATIONS}

The Navier-Stokes equations in conservative form, given by

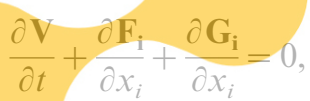

(1)

where, for $2 \mathrm{D}$ cases

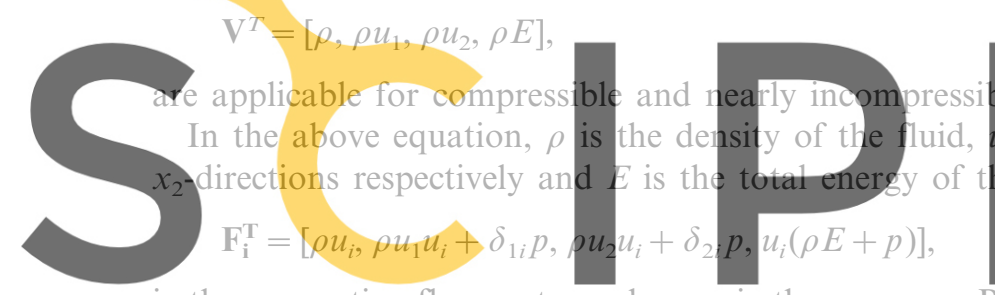

is the convective flux vector, where $p$ is the pressure. Both pressure and total energy can be defined by the set given in Equation. (2) and the state law. The diffusive flux vector is Register for free at https//WwW, scipedia.com to download the version withc
\[ \mathrm{G}_{\mathrm{i}}^{\mathrm{T}}=\left[0,-\tau_{1 i},-\tau_{2 i},-k \frac{\partial T}{\partial x_{i}}+\tau_{i j} u_{j}\right] \]

Here, $T$ is the temperature, and $\tau$ is the shear stress tensor given by

$$
\tau_{i j}=\mu\left(\frac{\partial u_{i}}{\partial x_{j}}+\frac{\partial u_{j}}{\partial x_{i}}\right)-\frac{2}{3} \mu \frac{\partial u_{k}}{\partial x_{k}} \delta_{i j}
$$

where $\mu$ is the dynamic viscosity of the fluid. The equation of state is

$$
p=(\gamma-1) \rho\left(E-0.5 u_{i}^{2}\right) .
$$

The speed of sound is related to $p$ and $\rho$, as

$$
c^{2}=\frac{\gamma p}{\rho} .
$$

Finally, the temperature dependence of viscosity is usually incorporated through the Sutherland's semi-empirical formula, as 


$$
\frac{\mu}{\mu_{r}}=\frac{T_{r}+S_{o}}{T+S_{o}}\left(\frac{T}{T_{r}}\right)^{1.5},
$$

where $S_{o}$ is Sutherland's constant and is equal to $198.6^{\circ}$ Rankine.

\section{THE NUMERICAL SOLUTION PROCEDURE}

The general algorithm developed by Zienkiewicz and Codina [16] is used in the present study for solving compressible flow problems. There are many advantages in using this algorithm. The characteristic type of approach leads to consistent upwinding terms which automatically control the stability $[14,17,18]$ in highly convective regions. Also, the generalised form of the approach can be used for both compressible and incompressible flows. Finally, the sound mathematical basis of the present scheme justifies its usage in all areas of fluid mechanics.

Before going into the details of the shock capturing techniques, it is essential to summarise the steps in the general algorithm. In the first step, intermediate values of conservative variables of the momentum equations are calculated omitting the pressure terms. In the second step, the equation of continuity is solved to determine the density changes in the fluid. The pressure is calculated from the density field using the gas law. Finally, the approximate values of the momentum components are corrected using the computed pressure values. These steps are discretized using the characteristic Galerkin method described in detail in Zienkiewicz and Codina [16]. The steps for the explicit form are

Step 1
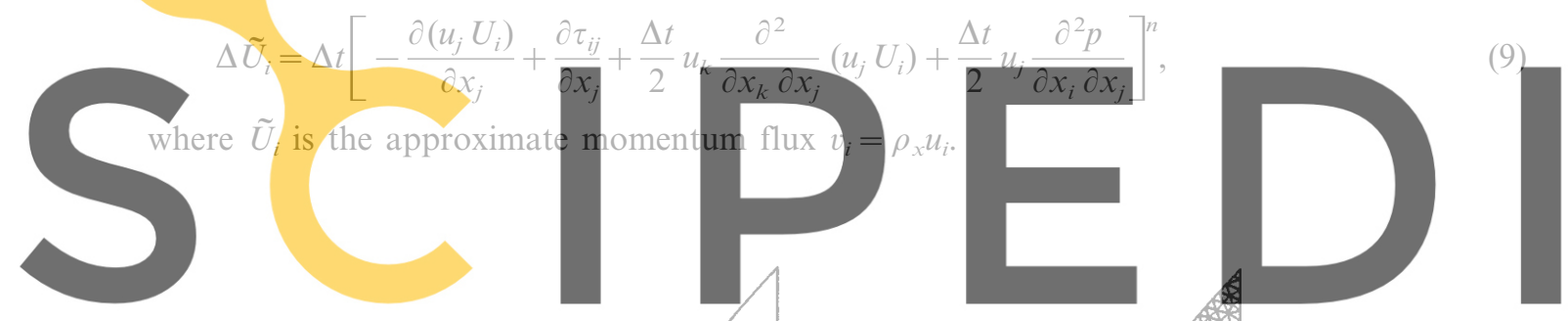

Register for free at https//www.scipedia.com to download the version without the

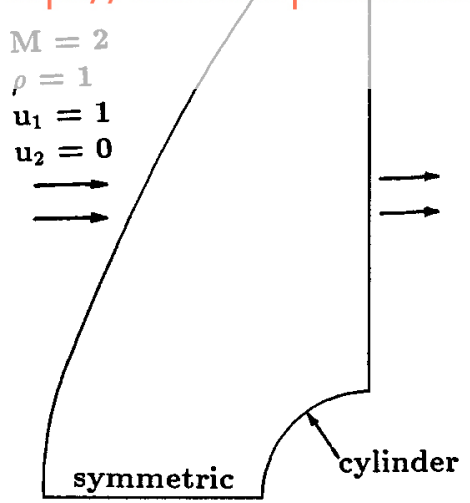

(a)

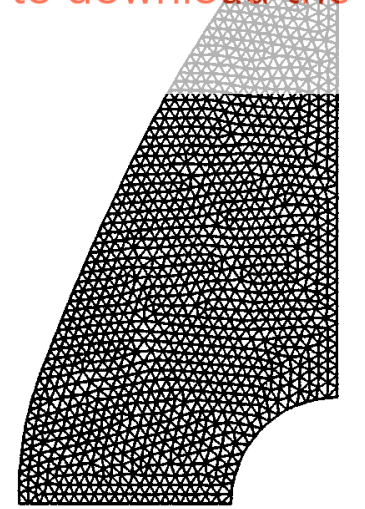

(b)

Figure 1. Supersonic inviscid flow past a quarter cylinder $(M=2)$. (a) Problem domain and boundary conditions; (b) finite element mesh (nodes $=1226$, elements $=2294)$. 

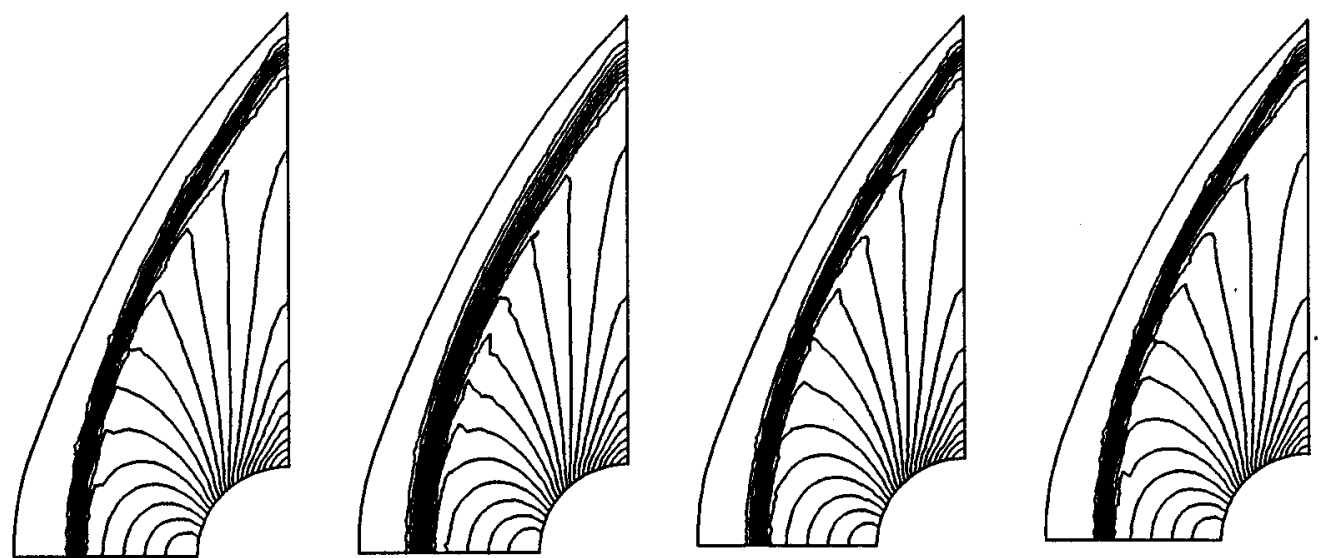

II

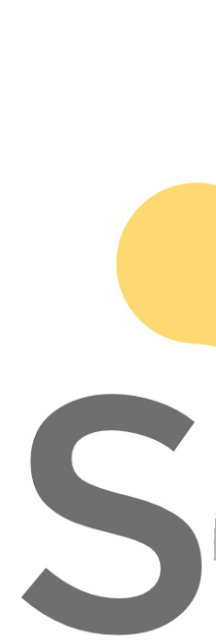

I

(a)

III

IV
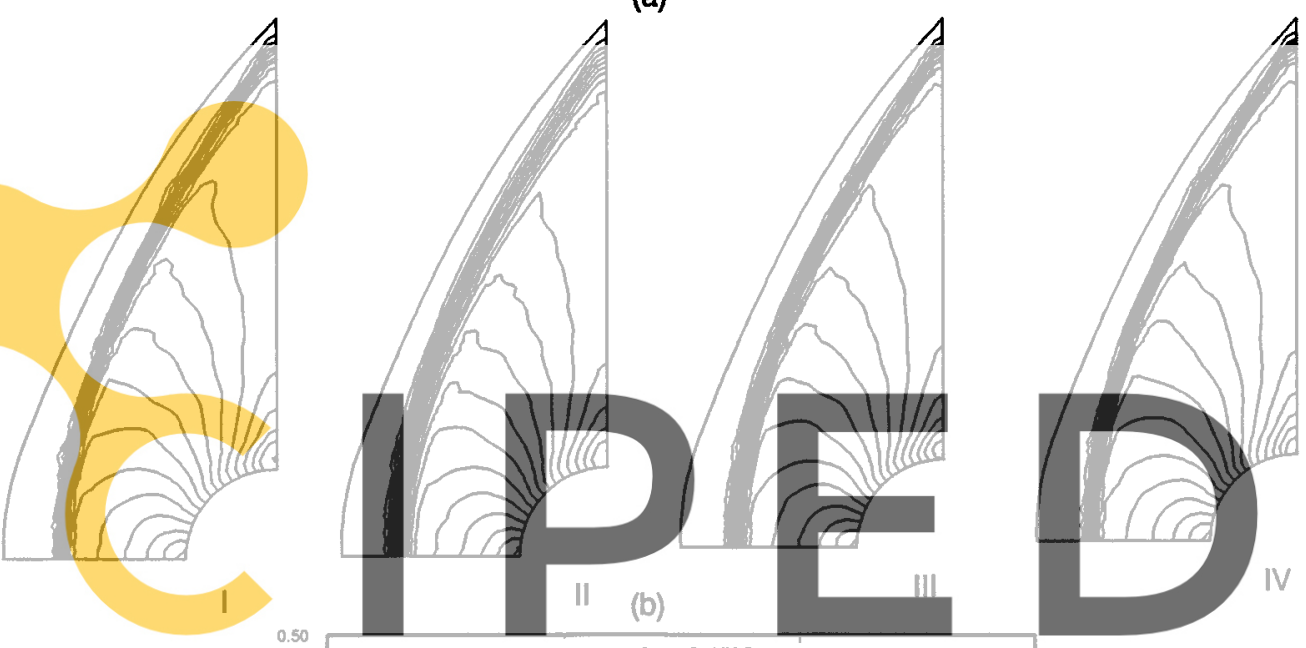

Method IV

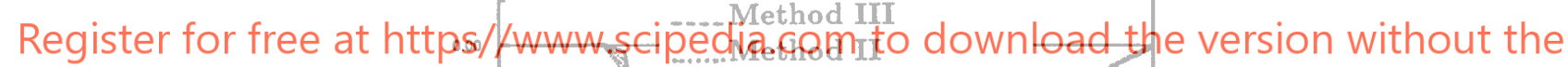

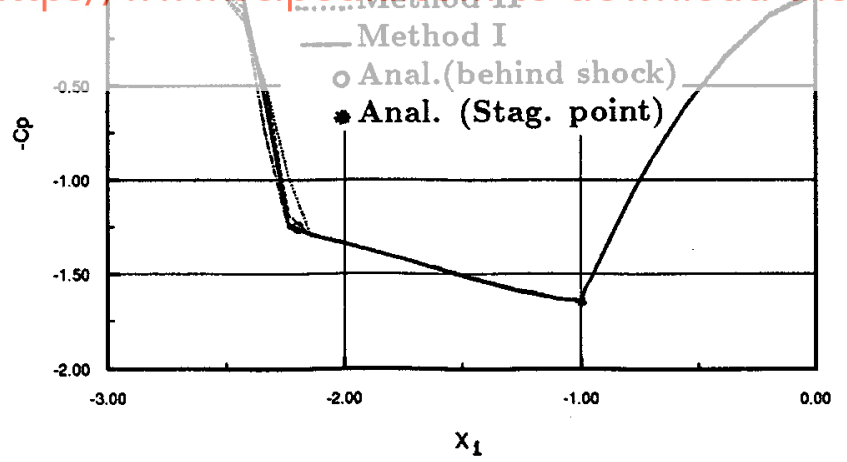

(c)

Figure 2. Results obtained for supersonic inviscid flow past a quarter cylinder. (a) Pressure contours; (b) Mach number contours; (c) comparison of coefficient of pressure in $x_{1}$-direction. 


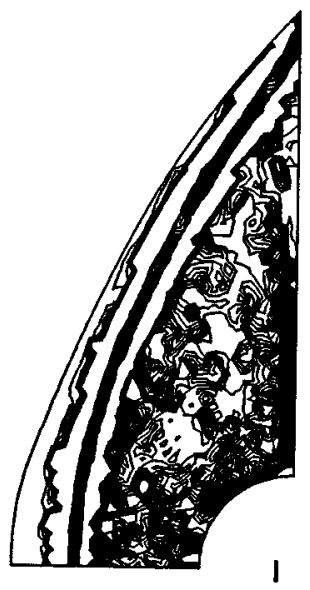

I

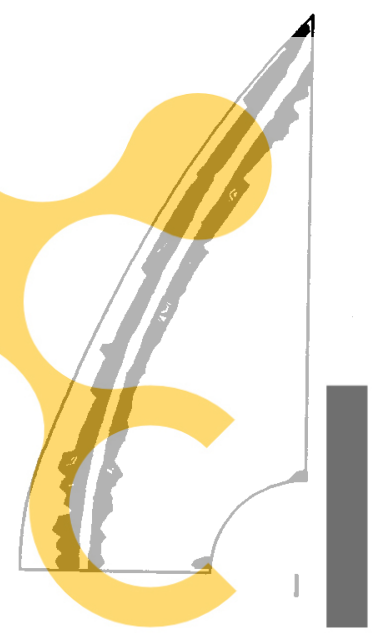

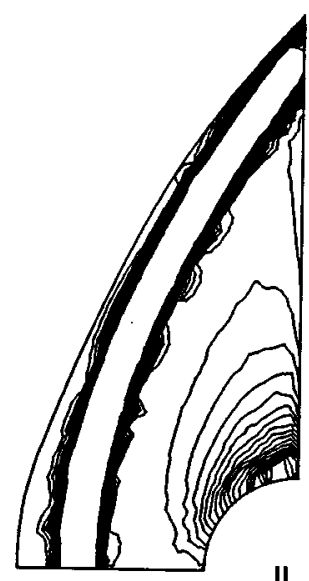

(a)

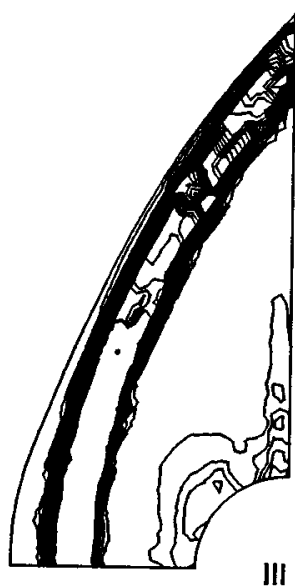

III
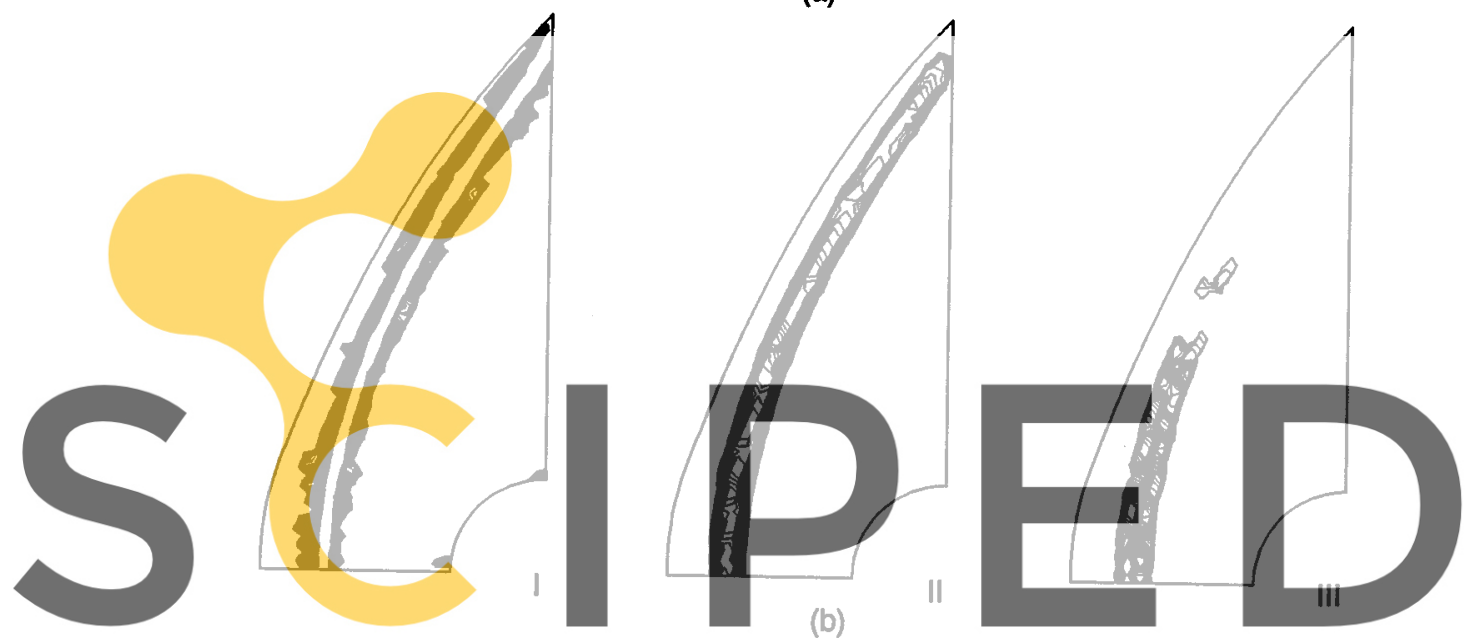

Register for free at https//www.scipedia com to dowpload the version without the
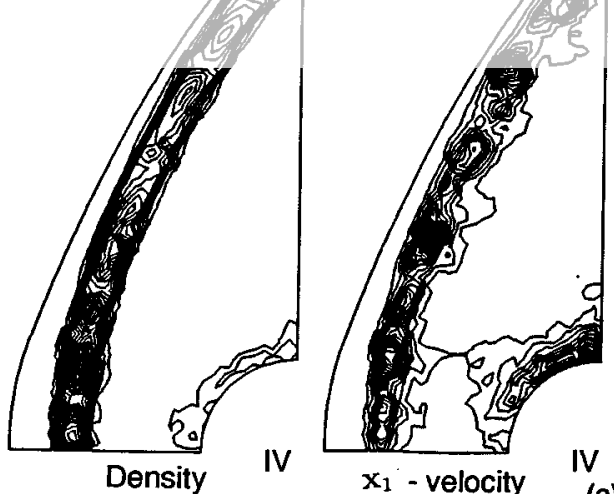

(c)
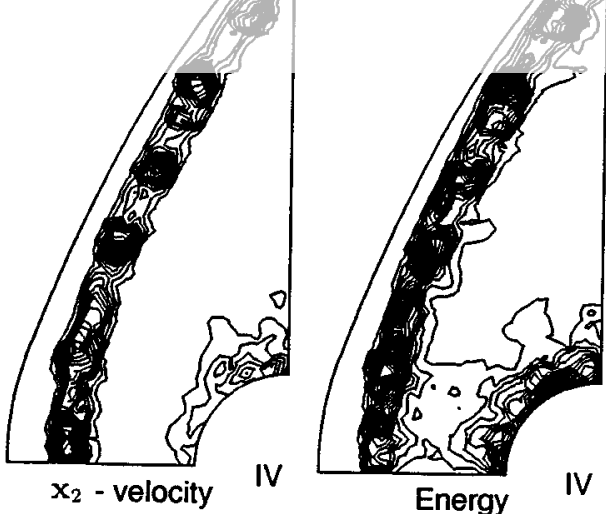

Figure 3. (a) Shock capturing viscosity coefficient distribution for methods I, II and III (0.0-0.5); (b) shock capturing viscosity coefficient distribution for methods I, II and III (0.5-1.0); (c) spatial residual distribution for method IV. 
Step 2

$$
\Delta \rho=-\Delta t\left[\frac{\partial U_{i}}{\partial x_{i}}+\theta_{1} \frac{\partial \Delta \tilde{U}_{i}}{\partial x_{i}}-\Delta t \theta_{1} \frac{\partial^{2} p}{\partial x_{i} \partial x_{i}}\right]^{n} .
$$

Step 3

$$
\Delta U_{i}=\Delta \tilde{U}_{i}-\Delta t\left[\frac{\partial p}{\partial x_{i}}\right]^{n} .
$$

Step 4

$$
\begin{aligned}
\Delta(\rho E)= & -\Delta t\left[\frac{\partial}{\partial x_{i}}\left(u_{i}(\rho E+p)\right)-\frac{\partial}{\partial x_{i}}\left(k \frac{\partial T}{\partial x_{i}}\right)+\frac{\partial}{\partial x_{i}}\left(u_{i} p\right)-\frac{\partial}{\partial x_{i}}\left(\tau_{i j} u_{j}\right)\right]^{n} \\
& +\left[\frac{\Delta t^{2}}{2} u_{k} \frac{\partial^{2}}{\partial x_{k} \partial x_{k}}\left(u_{i}(\rho E+p)\right)\right]^{n},
\end{aligned}
$$

where $\theta_{1}=1$ in Equation (10). The variables are defined in the previous section. The weak form and detailed solution procedure are discussed in Reference [14]. In this paper, linear triangular elements are used.

\section{SHOCK CAPTURING VISCOSITIES}

Although Equations (9)-(12) are stable in smooth flow regimes, they admit oscillations in the vicinity of shocks. To smooth such local effects, additional shock capturing viscosity has to be introduced. This is accomplished by means of an additional step in which smoothed values are computed according to a relationship $\phi_{s}^{n+1}=\phi^{n+1}+\Delta t \operatorname{div}\left(\mu_{a} \operatorname{grad} \phi\right.$ where $\mu_{a}$ denotes an additional artifici pidus [7] uses an artificial alternative model due to MacCormack and Baldwin [s
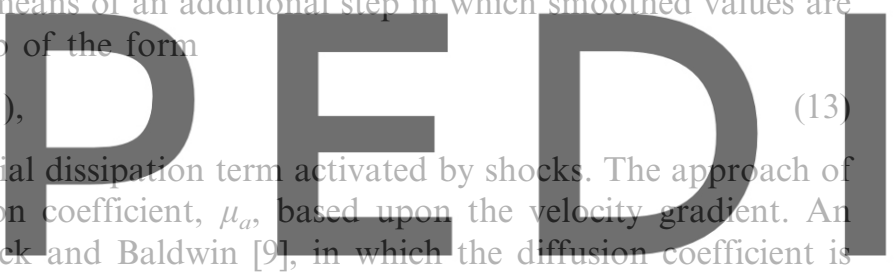

based upon second derivative of pressure, however, gives better results [12]. Nodal values of

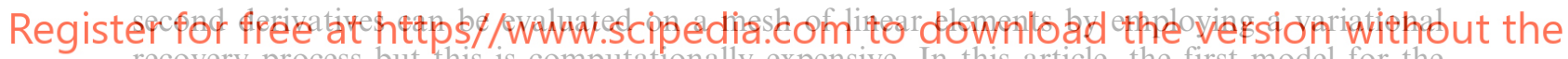
recovery process but this is computationally expensive. In this article, the first model for the artificial diffusion is the modified form of MacCormack-Baldwin scheme which is slightly cheaper than the original method. Here the second gradient is approximately calculated from the consistent and lumped mass matrices.

The model is derived from the observation that in one-dimension, for a scalar variable $p$ we can write (see Reference [12])

$$
\left.\frac{\partial}{\partial x_{i}}\left(h^{2} \frac{\partial p}{\partial x_{i}}\right) x_{j} \approx M_{\mathrm{L}}^{-1}\left(M-M_{\mathrm{L}}\right) \bar{p}\right|_{j}
$$

where $M$ denotes the consistent mass matrix and $M_{\mathrm{L}}$ the diagonal lumped mass matrix. The expression appearing on the right of the above equation obviously produces a diffusion effect and we use this fact as the basis of the construction of the artificial viscosity model. In two-dimensions, the relation

$$
\mu_{a}=\frac{C_{e} S_{e}}{\Delta t_{e}}
$$



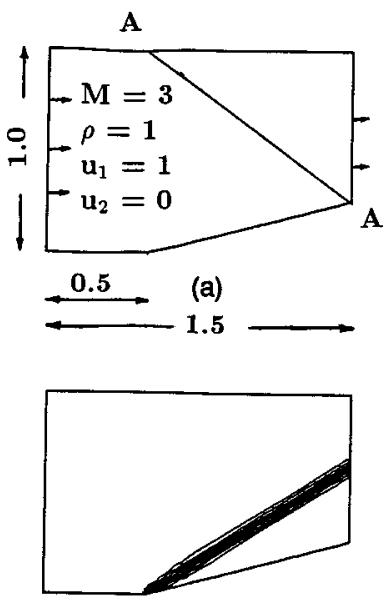

I
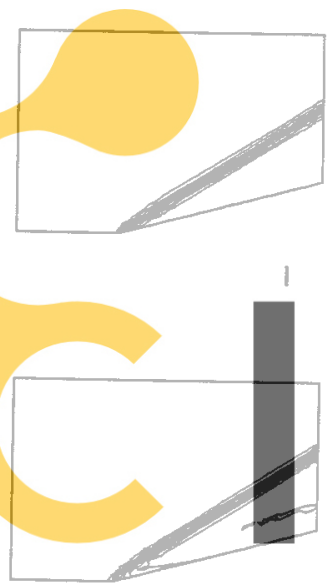

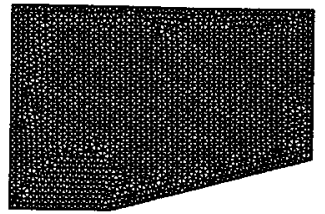

(b)

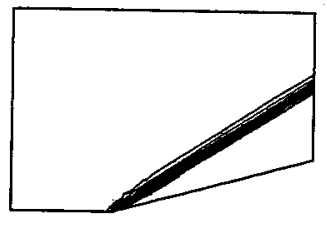

III

(d)

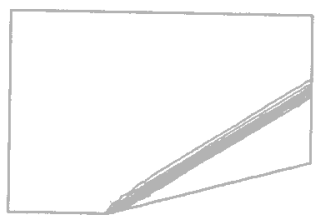

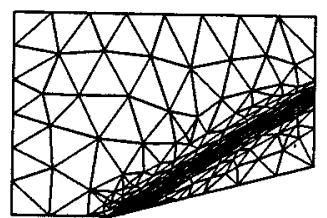

(c)

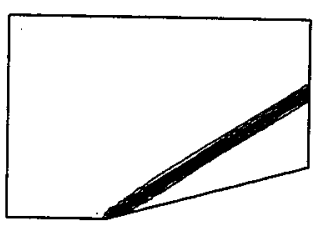

IV

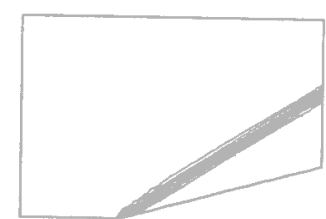

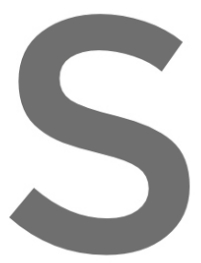
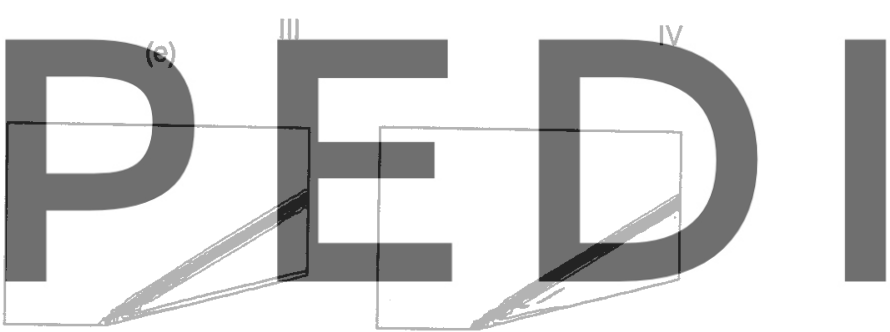

\section{Register for free at https//www.scipedia.६pm tandownload the version without the}

Figure 4. Supersonic inviscid flow past a $15^{\circ}$ wedge $(M=3)$. (a) Geometry and boundary condition; (b) uniform grid (nodes $=1780$, elements $=3396$ ); (c) adapted mesh (nodes =168, elements =286); (d) density contours; (e) pressure contours; and (f) Mach number contours for the uniform mesh.

is used in the MacCormack-Baldwin scheme [12]. The element time step is included to ensure that the added diffusion is conservative at steady state. The element 'pressure switch' $S_{e}$ is taken to be the mean of the element nodal switches, $S_{i}$, which are computed as

$$
S_{i}=\frac{\left|\sum_{e}\left(p_{i}-p_{k}\right)\right|}{\sum_{e}\left|p_{i}-p_{k}\right|} .
$$

It can be verified that $S_{i}=1$ when pressure has a local extremum at node $i$ and $S_{i}=0$ when pressure at node $i$ is the average of values for all nodes adjacent to node $i . C_{e}$ is a user specified constant, normally ranging from 0.0 to 2.0 . 

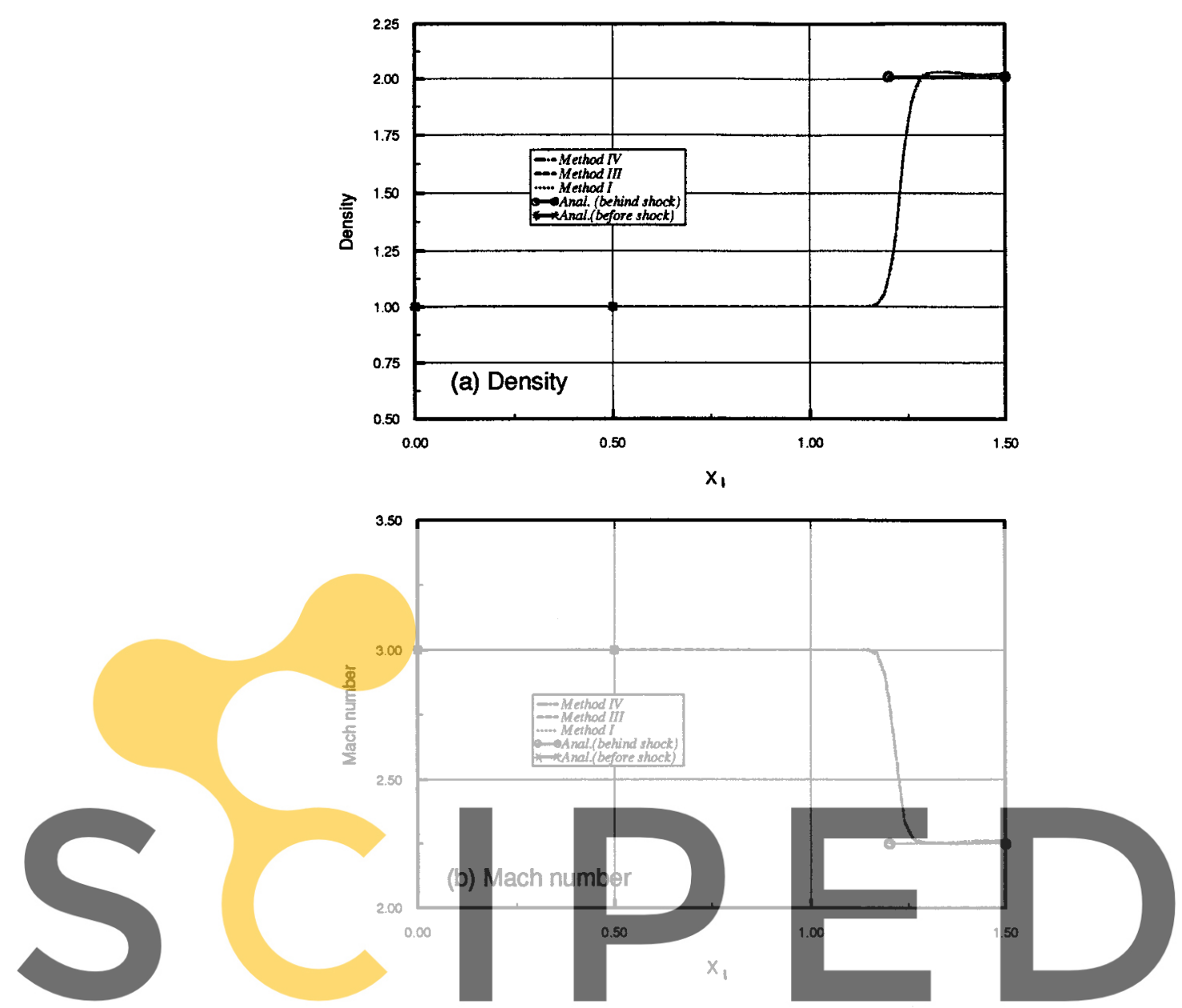

Register for free at https ${ }_{2.50}^{3.00} /$ www.scipedia.com to download the version without the

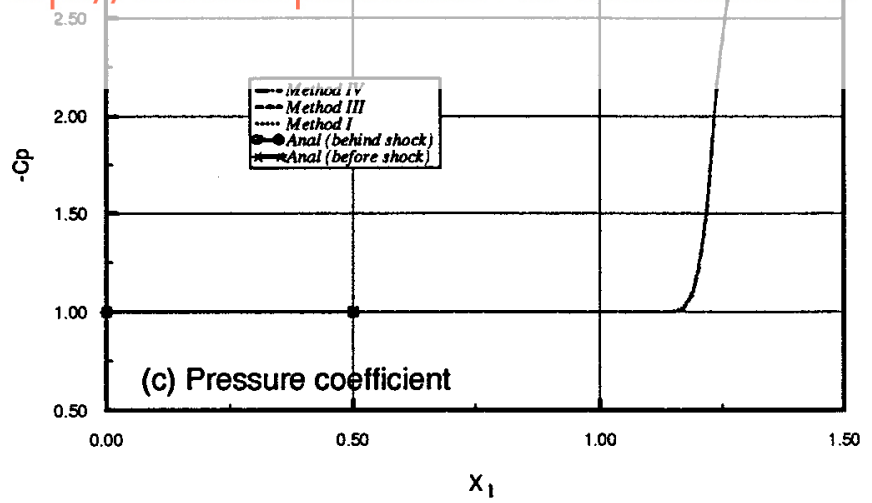

Figure 5. Comparison of density, Mach number and pressure coefficient along section AA for the uniform mesh. 

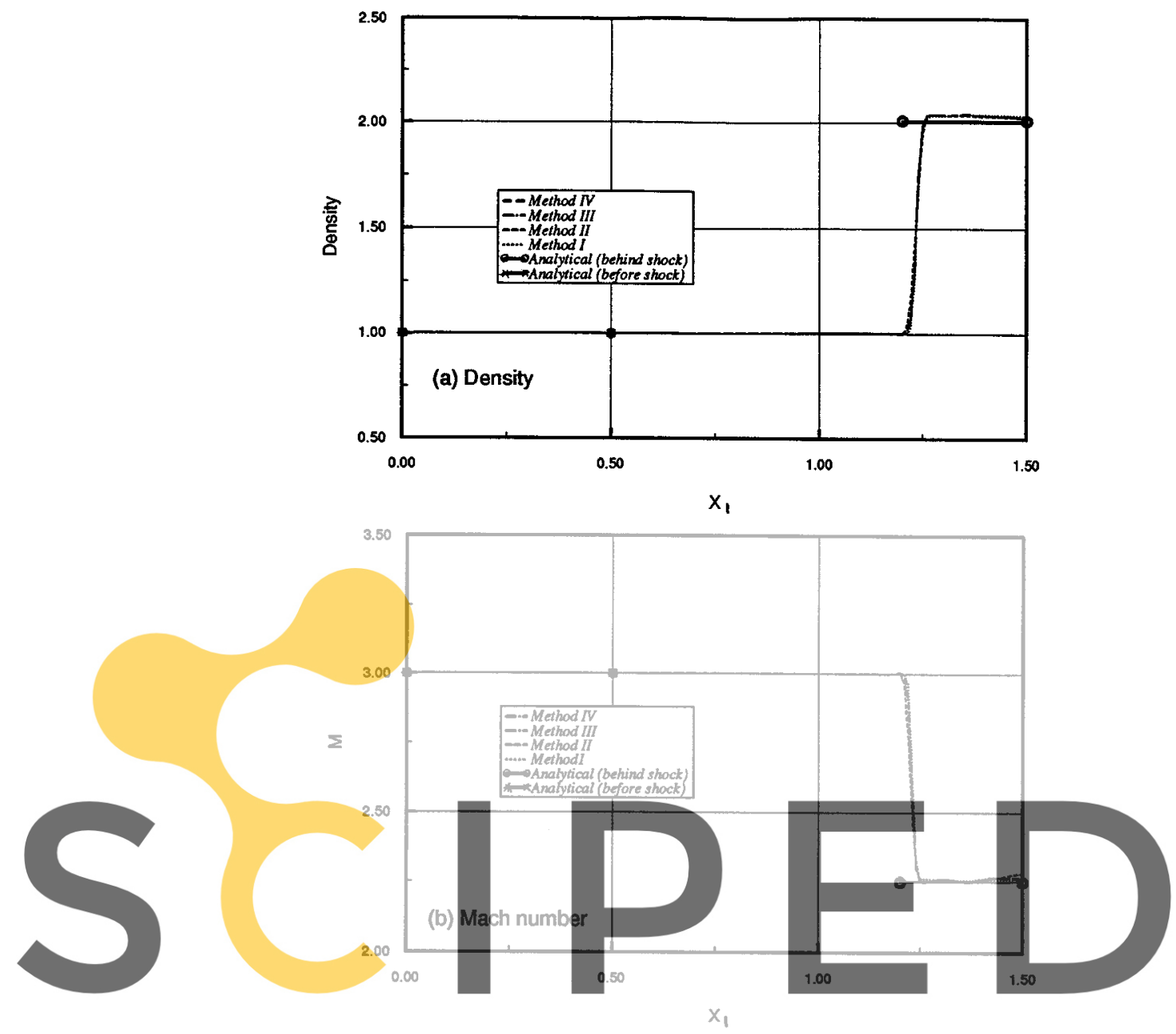

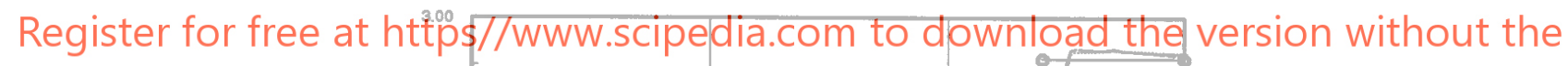

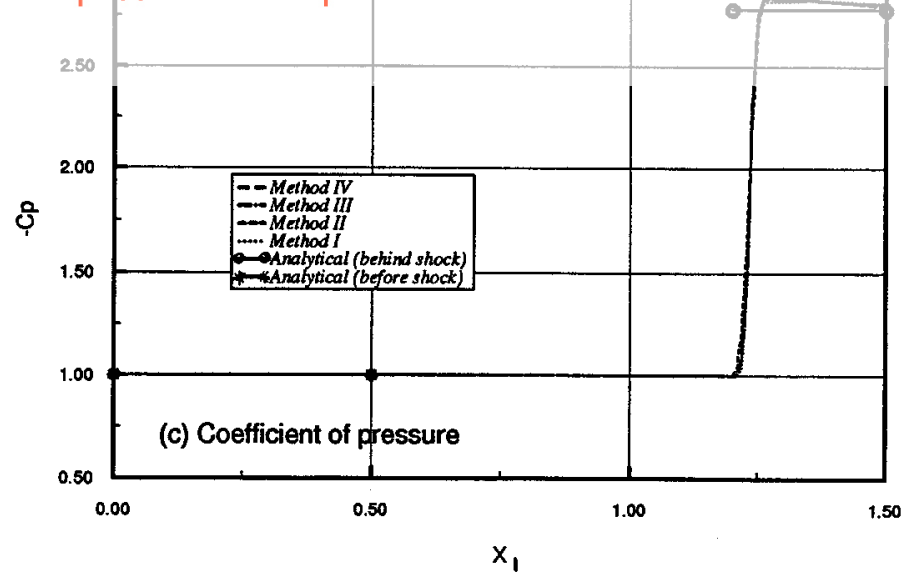

Figure 7. Comparison of present predictions in adapted mesh with the analytical solution. 


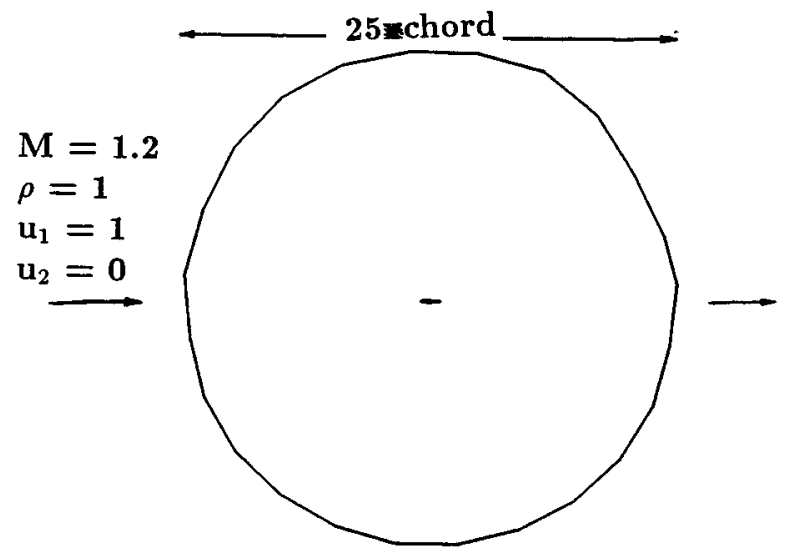

(a)

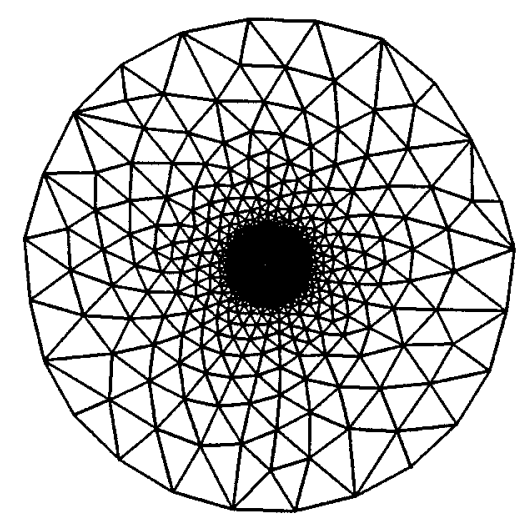

(b)

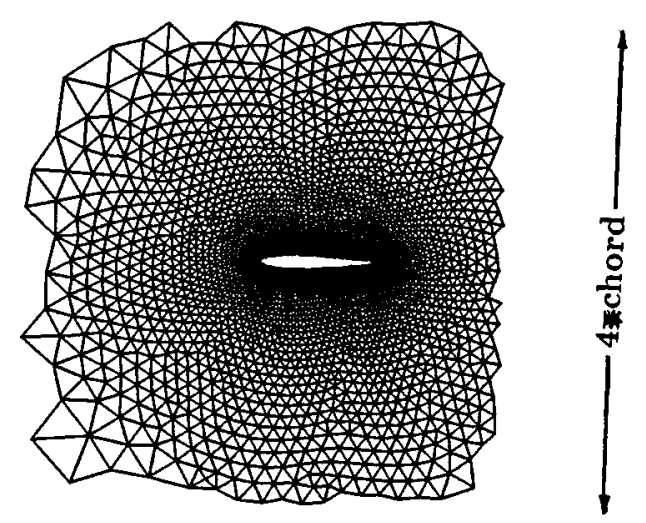

(c)

Figure 8. Supersonic inviscid flow past a NACA0012 aerofoil $(M=1.2)$. (a) Problem formulation; (b) finite element mesh $($ nodes $=3753$, elements $=7351)$; (c) mesh near the aerofoil surface. 


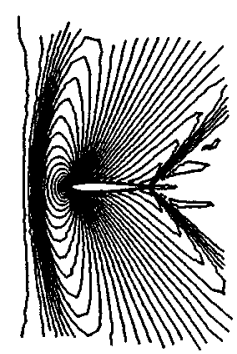

1

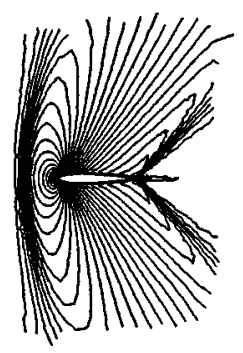

I

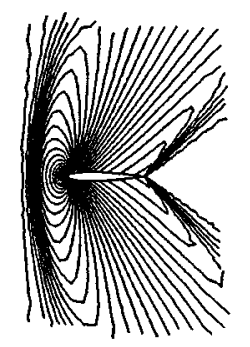

I

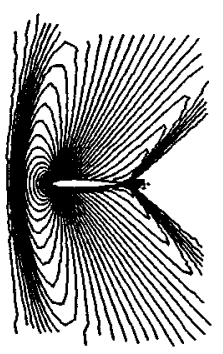

II

(a)

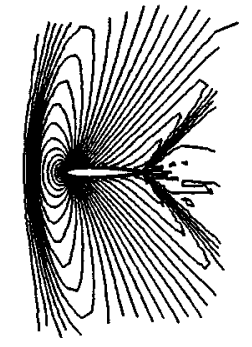

II

(b)

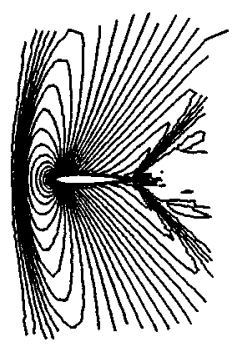

III

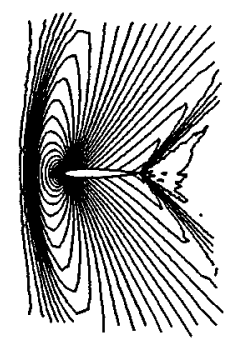

II

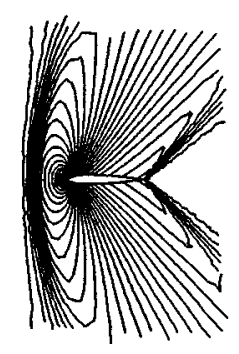

III

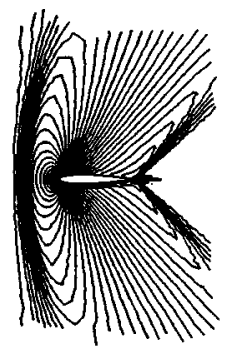

III

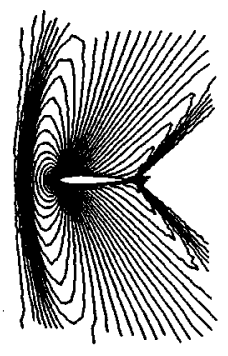

IV

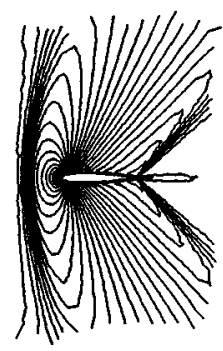

IV

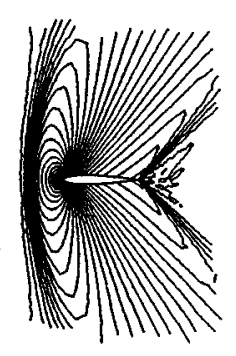

IV

(c)

Figure 9. Results obtained for supersonic inviscid flow past an aerofoil. (a) Density contours; (b) Mach number contours; and (c) pressure contours.

$$
\left[\frac{\phi_{s}^{n+1}-\phi^{n+1}}{\Delta t}\right]=\boldsymbol{M}_{\mathrm{L}}^{-1} C_{e} h^{2}|\nabla V|_{e} \int_{\Omega} \frac{\partial \boldsymbol{N}^{T}}{\partial x_{i}} \frac{\partial \boldsymbol{N}}{\partial x_{i}} \phi^{n},
$$

where $V$ is the total velocity vector.

In the later studies, however, the dissipation based on pressure was proved to be the most efficient for shock capture [12]. A method similar to that of Lapidus replacing the gradient of velocity by the pressure gradient is another shock capturing viscosity method (method II) examined in this article. The following relation is used to add artificial diffusion:

$$
\left[\frac{\phi_{s}^{n+1}-\phi^{n+1}}{\Delta t}\right]=\boldsymbol{M}_{\mathrm{L}}^{-1} C_{e} h^{2} \frac{|\boldsymbol{V}|+c}{\bar{p}}|\nabla p|_{e} \int_{\Omega} \frac{\partial \boldsymbol{N}^{T}}{\partial x_{i}} \frac{\partial \boldsymbol{N}}{\partial x_{i}} \phi^{n}
$$

Here the first gradient of pressure is calculated at element level from the nodal pressure values, as 


$$
|\nabla p|_{e}=\sqrt{\left(\frac{\partial \boldsymbol{N}}{\partial x_{1}} p_{i}^{h}\right)^{2}+\left(\frac{\partial \boldsymbol{N}}{\partial x_{2}} p_{i}^{h}\right)^{2}},
$$

where $\boldsymbol{N}$ is the shape function and $p^{h}$ is the finite element solution.

Finally, the anisotropic type of shock capturing [15,16] (method IV) is examined and compared with other schemes. The basic idea of this method is to introduce an anisotropic additional diffusion to discrete equations proportional to the spatial residual of the previous time step, thus keeping the consistency of the finite element formulation when the steady state is reached. The expression for the corrected solution is given for any variable except density (diffusion is added to the density equation isotropically), and is

$$
\begin{aligned}
\frac{\phi_{s}^{n+1}-\phi^{n+1}}{\Delta t}= & v_{s c}\left(\frac{\partial^{2} \phi}{\partial x_{1}^{2}}+\frac{\partial^{2} \phi}{\partial x_{2}^{2}}\right) \\
& -v_{c g}\left[\frac{u_{1}^{2}}{V^{2}} \frac{\partial^{2} \phi}{\partial x_{1}^{2}}+\frac{u_{2}^{2}}{V^{2}} \frac{\partial^{2} \phi}{\partial x_{2}^{2}}+\frac{u_{1} u_{2}}{V^{2}} \frac{\partial}{\partial x_{1}}\left(\frac{\partial \phi}{\partial x_{2}}\right)+\frac{u_{2} u_{1}}{V^{2}} \frac{\partial}{\partial x_{2}}\left(\frac{\partial \phi}{\partial x_{1}}\right)\right] .
\end{aligned}
$$

In the above equation, $v_{s c}$ is the total additional viscosity, $v_{c g}$ is the amount subtracted from the total additional viscosity and $V$ is the total velocity vector. The second term in the right-hand-side of the above equation is equal to the additional diffusion added by the characteristic Galerkin scheme. The following expressions are used for the $v_{s c}$ and $v_{c g}$ in the present study.

$$
\boldsymbol{v}_{s c}=\frac{1}{2} C_{e} h \frac{|\boldsymbol{R}|}{|\nabla \phi|},
$$

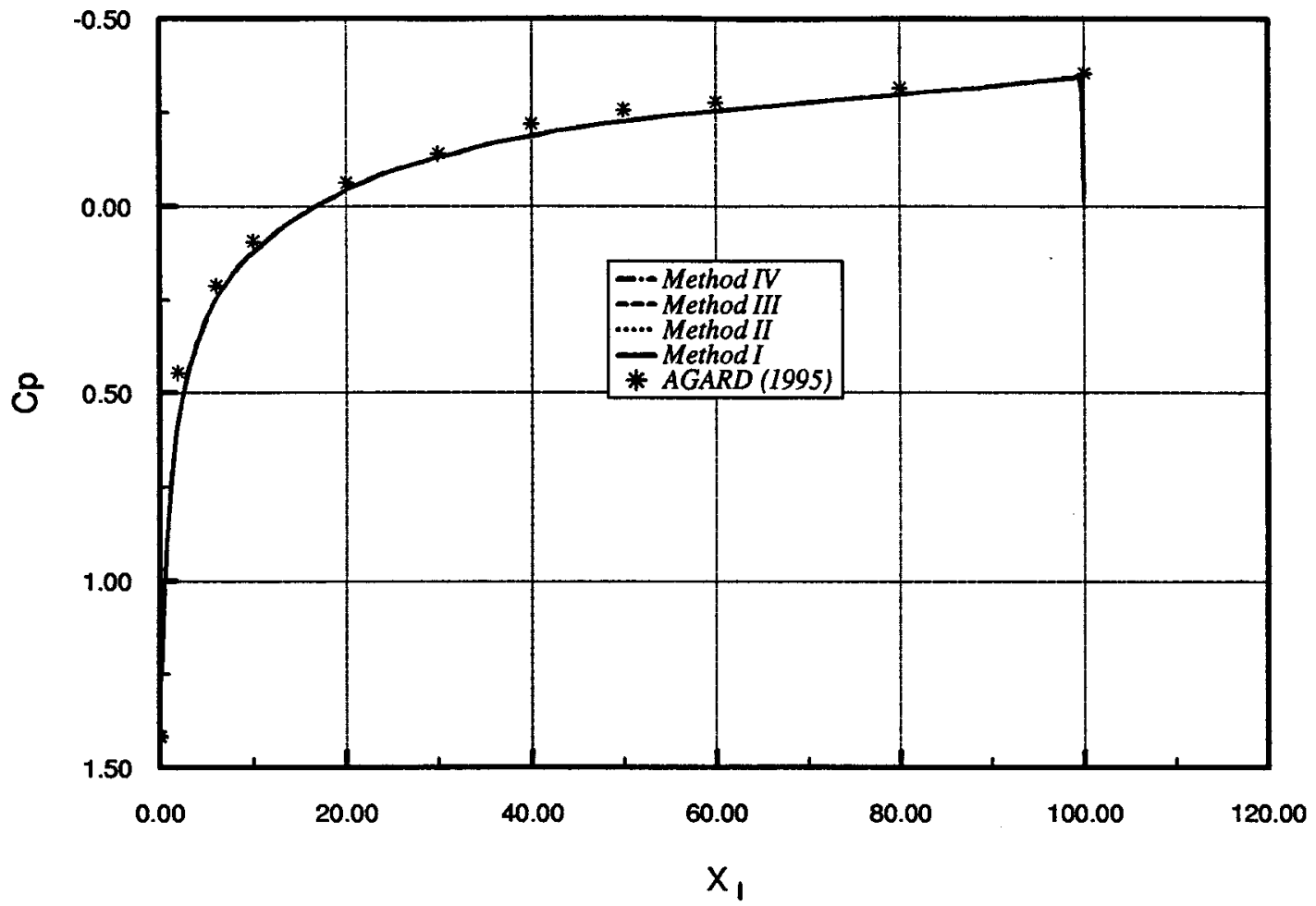

Figure 10. Comparison of surface pressure coefficient distribution with literature. 


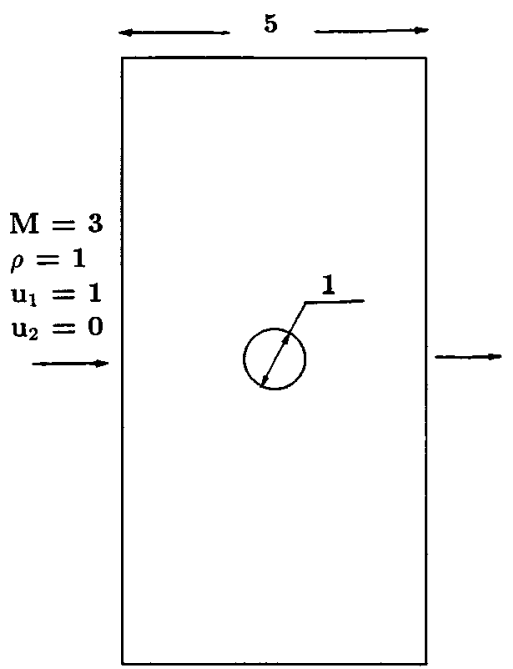

(a)

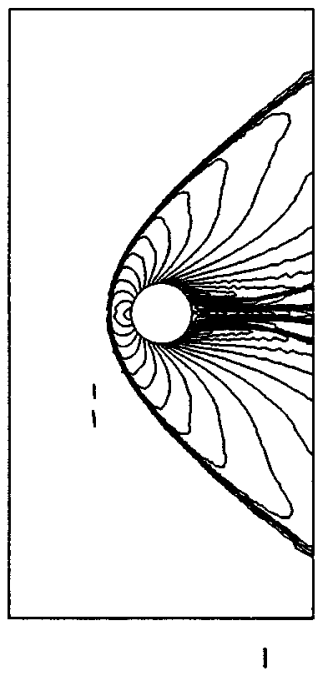

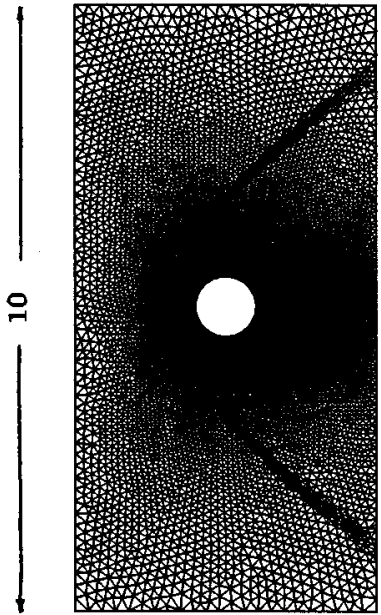

(b)

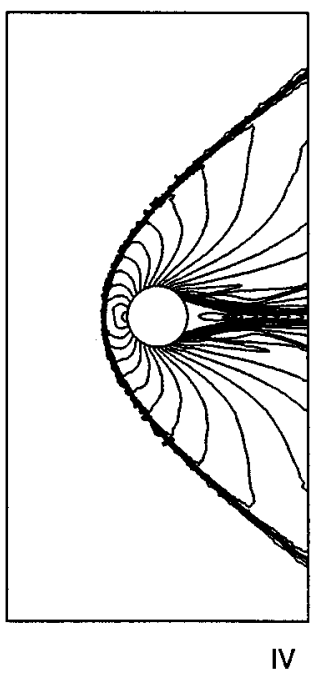

(c)

Figure 11. Supersonic inviscid flow past a full cylinder $(M=3)$. (a) Problem formulation; (b) adapted finite element mesh $($ nodes $=12651$, elements $=24979$ ); and $(c)$ Mach contours.

and

$$
\boldsymbol{v}_{c g}=\frac{1}{2} \frac{\Delta t}{2} \boldsymbol{V}^{2}
$$

where $R_{i}$ is the spatial residual calculated without the upwinding terms for the variables, $h$ is the characteristic dimension of the triangular elements, $C_{e}$ is the user specified constant. 


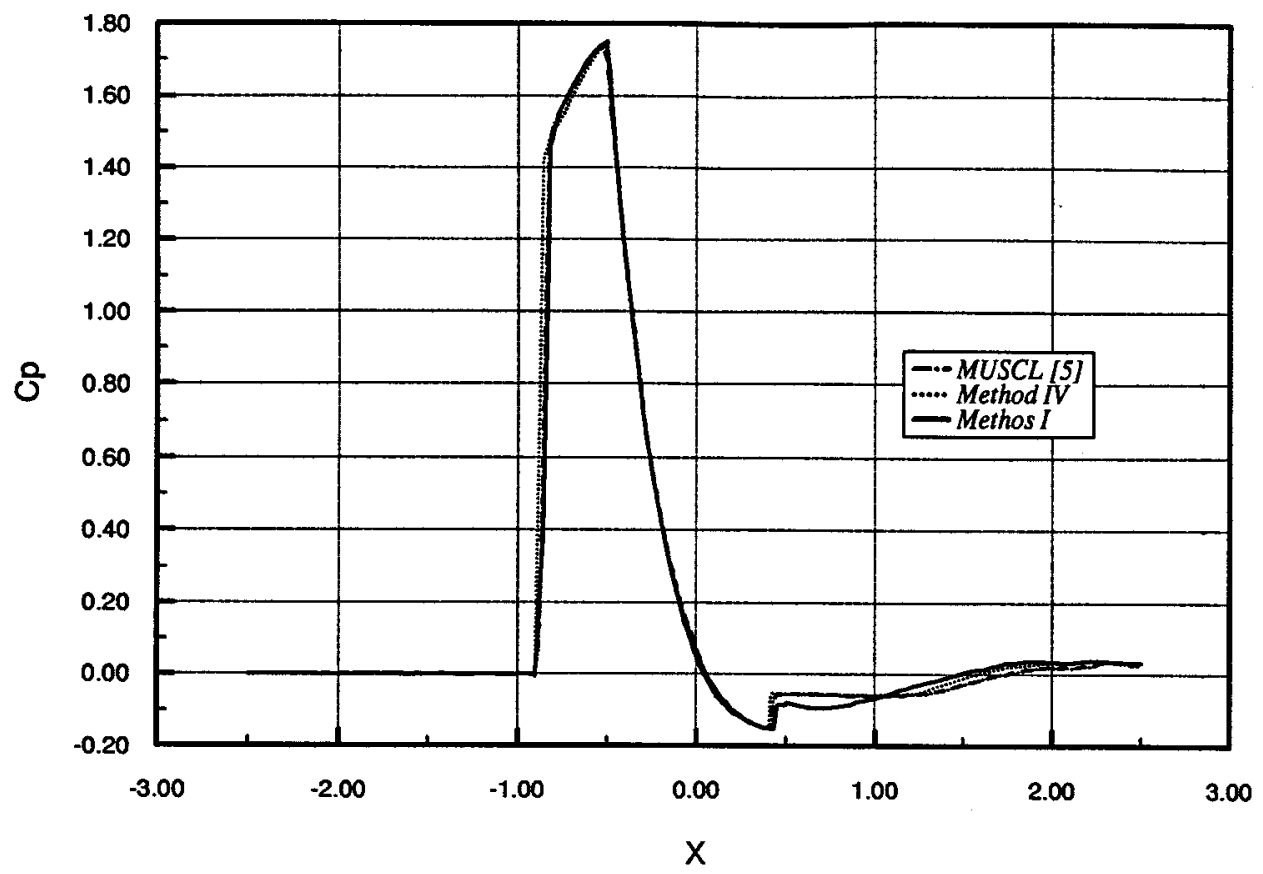

(a)

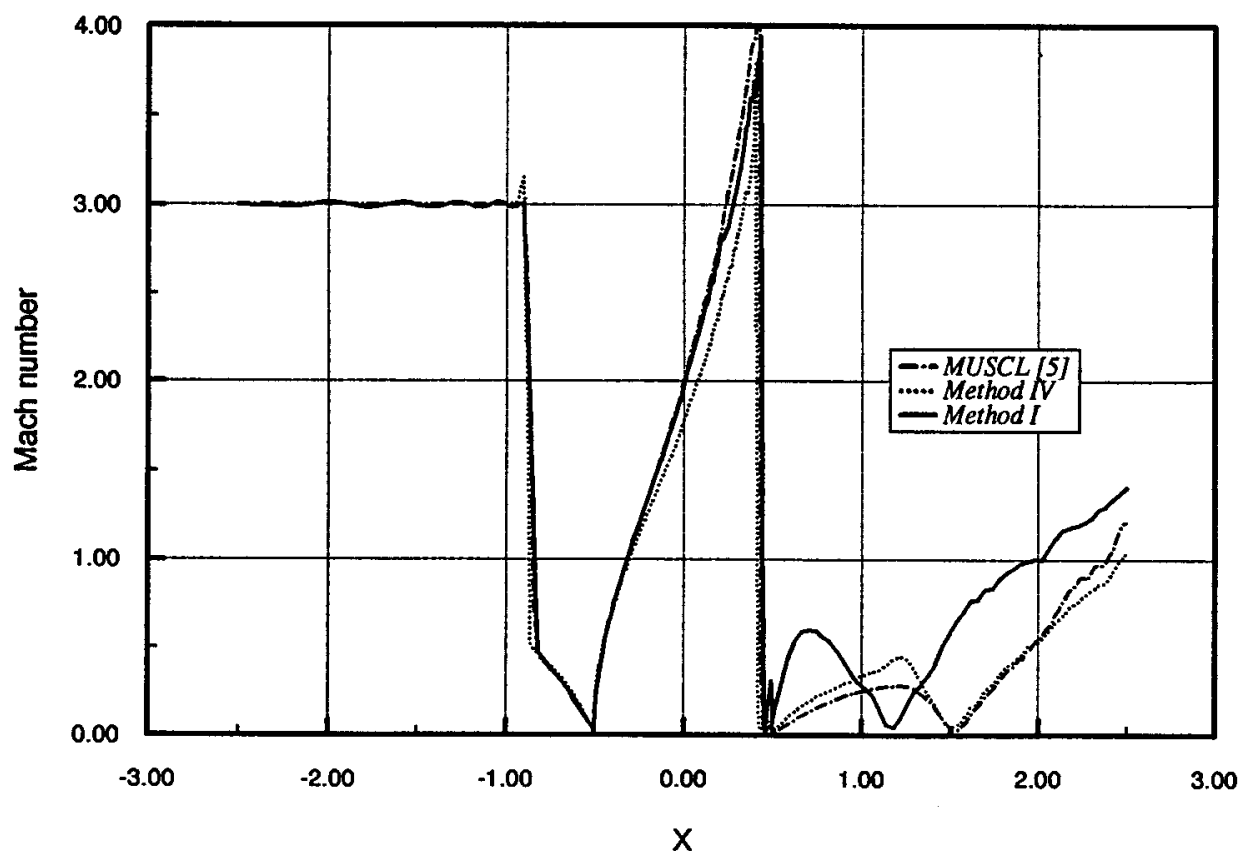

(b)

Figure 12. Property variation along the mid-height. (a) Coefficient of pressure; and (b) Mach number. 
Equation (21) can be rewritten with indices as

$$
\frac{\phi_{s}^{n+1}-\phi^{n+1}}{\Delta t}=\frac{\partial}{\partial x_{i}}\left[\delta_{i j} v_{s c} \frac{\partial \phi}{\partial x_{j}}+\left(v_{s l}-v_{s c}\right)\left(\frac{u_{i} u_{j}}{\boldsymbol{V}^{2}}\right) \frac{\partial \phi}{\partial x_{j}}\right],
$$

where

$$
\boldsymbol{v}_{s l}=\max \left(0, \boldsymbol{v}_{s c}-\boldsymbol{v}_{c g}\right) .
$$

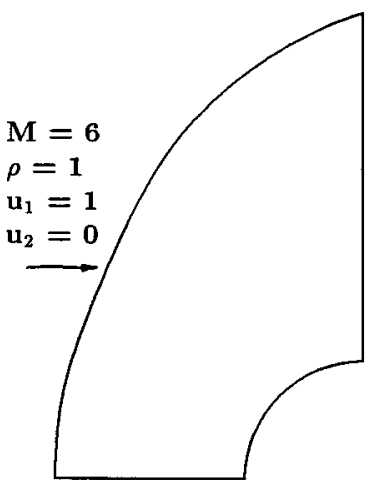

(a)

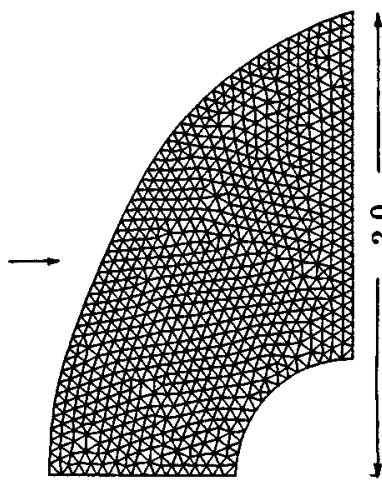

(b)

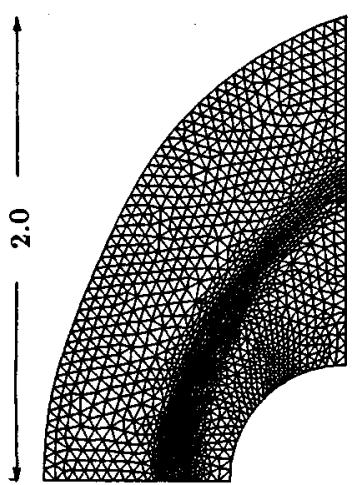

(c)
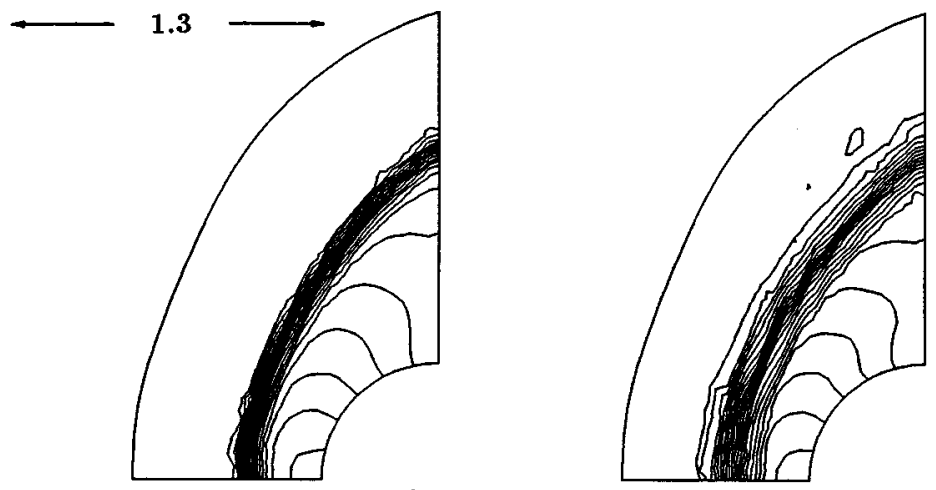

(d)

III

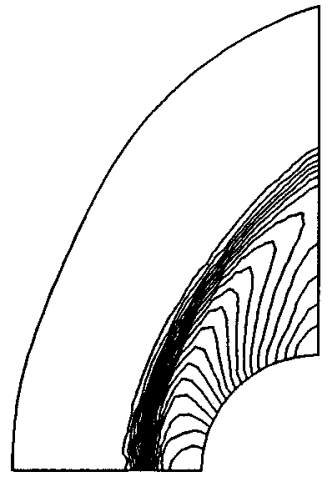

I

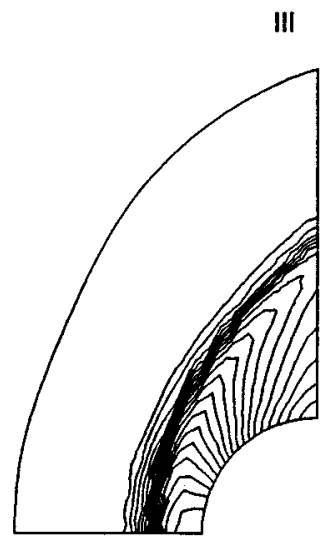

III

(e)

Figure 13. Hypersonic inviscid flow past a quarter cylinder $(M=6)$ (a) Problem formulation; (b) finite element mesh (nodes $=785$, elements $=1455$ ); (c) first adapted mesh (nodes $=1391$, elements $=2637$ ); (d) Mach number contours; and (e) pressure contours using uniform mesh. 
All the above described methods are implemented both for viscous and inviscid flow problems. The dissipation terms are added to the equations at every time step as the solution proceed towards steady state. For instance, method III described above is added to the momentum equation as

$$
\begin{aligned}
\Delta \tilde{U}_{i}= & \Delta t\left[-\frac{\partial\left(u_{j} U_{i}\right)}{\partial x_{j}}+\frac{\partial \tau_{i j}}{\partial x_{j}}+\frac{\Delta t}{2} u_{k} \frac{\partial^{2}}{\partial x_{k} \partial x_{j}}\left(u_{j} U_{i}\right)+\frac{\Delta t}{2} u_{j} \frac{\partial^{2} p}{\partial x_{i} \partial x_{j}}\right]^{n} \\
& +\Delta t \frac{\partial}{\partial x_{i}}\left[C_{e} \frac{|V|+c}{\bar{p}}\left|\nabla^{2} p\right|_{e} \frac{\partial U_{i}^{n}}{\partial x_{i}}\right] .
\end{aligned}
$$

Finite element discretization of the above equation leads to the final form

$$
\begin{aligned}
{[\boldsymbol{M}]\left\{\Delta \tilde{U}_{i}\right\}=} & -\Delta t\left\{[\boldsymbol{C}]\left\{u_{j} U_{i}\right\}+[\boldsymbol{K}]\left\{U_{i}\right\}+\frac{\Delta t}{2} u_{k}\left[\boldsymbol{K}_{u}\right]\left\{u_{j} U_{i}\right\}+\frac{\Delta t}{2} u_{k}\left[\boldsymbol{K}_{p}\right]\{p\}\right\}^{n} \\
& +\Delta t C_{e} h^{3} \frac{|u|+c}{\bar{p}}\left|\nabla^{2} p\right|_{e}[\boldsymbol{K}] U_{i}^{n},
\end{aligned}
$$

where

$$
\begin{aligned}
& {[\boldsymbol{M}]=\int_{\Omega} \boldsymbol{N}^{T} \boldsymbol{N} \mathrm{d} \Omega,} \\
& {[\boldsymbol{C}]=\int_{\Omega} \boldsymbol{N} \frac{\partial \boldsymbol{N}^{T}}{\partial x_{i}} \mathrm{~d} \Omega,} \\
& {[\boldsymbol{K}]=\int_{\Omega} \frac{\partial \boldsymbol{N}^{T}}{\partial x_{i}} \frac{\partial \boldsymbol{N}}{\partial x_{i}} \mathrm{~d} \Omega,} \\
& {\left[\boldsymbol{K}_{u}\right]=\int_{\Omega} \frac{\partial \boldsymbol{N}^{T}}{\partial x_{k}} \frac{\partial \boldsymbol{N}}{\partial x_{j}} \mathrm{~d} \Omega,} \\
& {\left[\boldsymbol{K}_{p}\right]=\int_{\Omega} \frac{\partial \boldsymbol{N}^{T}}{\partial x_{k}} \frac{\partial \boldsymbol{N}}{\partial x_{i}} \mathrm{~d} \Omega .}
\end{aligned}
$$

All the shock capturing viscosities discussed are used to solve a variety of inviscid and viscous problems with shock. In the following section, numerical examples with different Mach numbers are presented.

\section{NUMERICAL EXAMPLES}

\subsection{Inviscid flow past a cylinder of Mach number 2}

Figure 1 shows the problem formulation (a) and the finite element mesh (b) generated. This problem is a standard test case used by many authors to validate the numerical schemes. In the present study, an inlet Mach number of 2 is assumed and the fluid is inviscid. A uniform mesh is generated (Figure 1(b)) with 1226 nodes and 2294 linear triangular elements. All four shock capturing viscosity schemes are separately used to solve this problem.

An efficient shock capturing viscosity should add the additional artificial diffusion in a rational way and spread the discontinuity over several computational elements without significantly affecting the solution in the uniform flow region. In many schemes, the amount of additional diffusion is controlled by an user specified constant $C_{e}$. Suitable selection of this constant gives oscillation-free accurate results. The amount of spreading of the discontinuity 

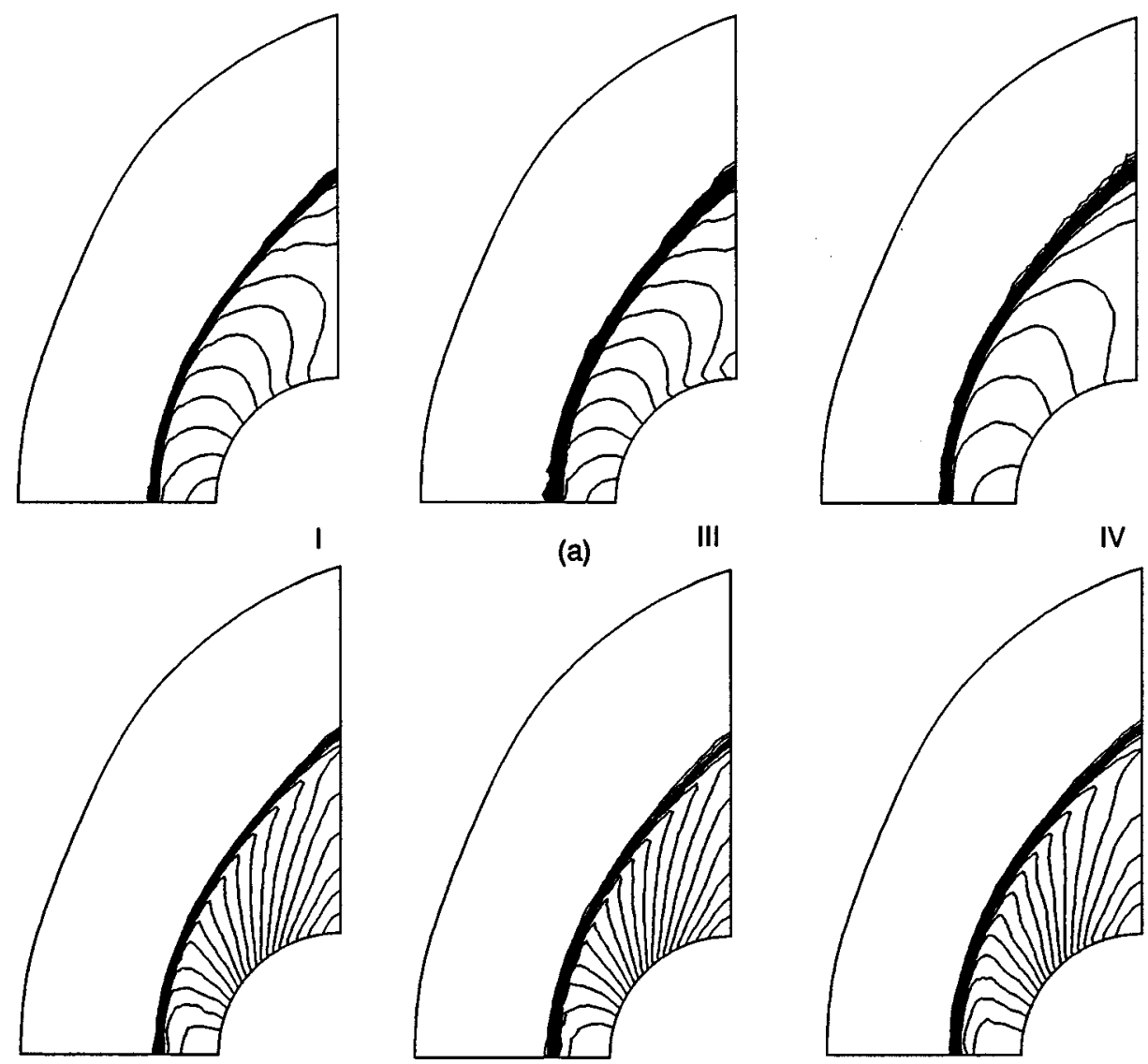

(a)

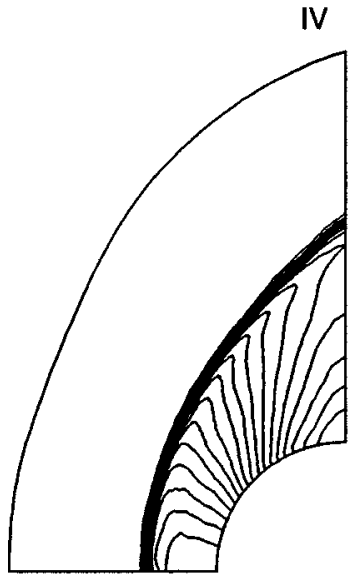

1

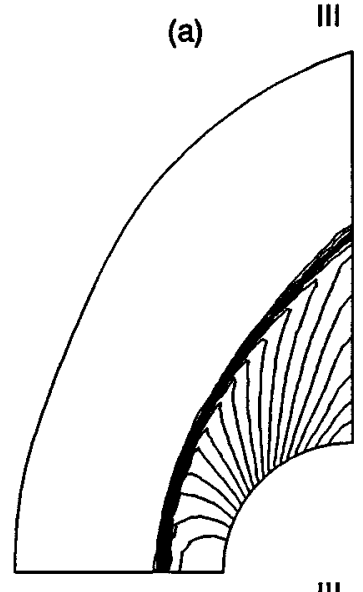

(b)

III

IV

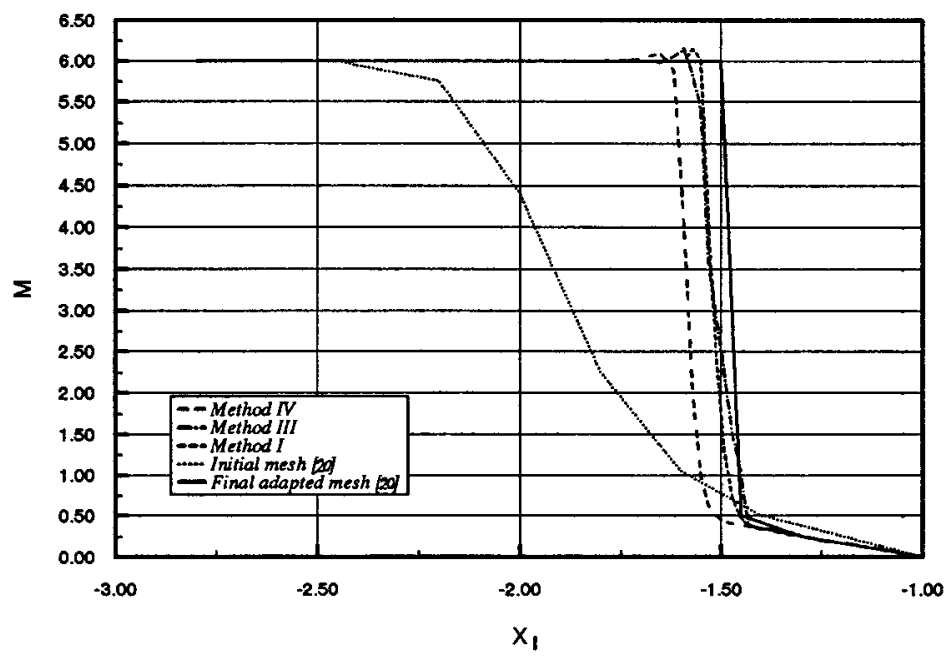

Figure 14. Results obtained for the hypersonic inviscid flow past quarter cylinder. (a) Mach number contours; and (b) pressure contours using adapted mesh; (c) comparison of Mach number along the bottom line in the $x_{1}$-direction. 

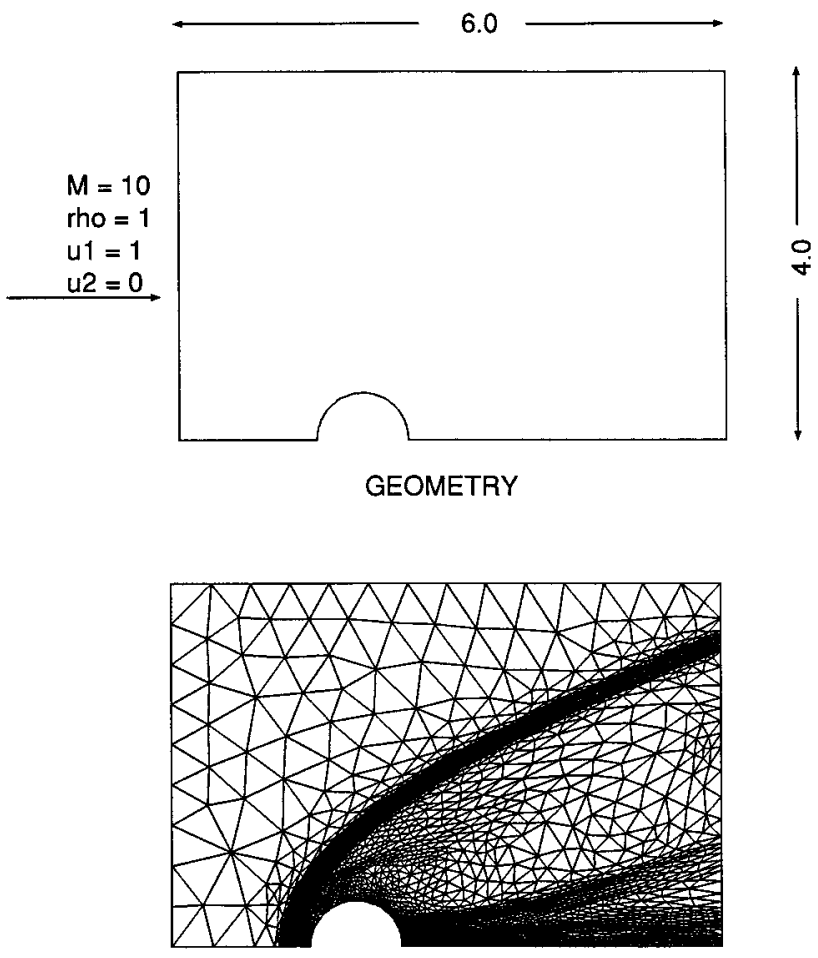

MESH, NODES $=4843$, ELEMENTS $=9441$

Figure 15. Hypersonic inviscid flow past a half cylinder $(M=10)$. (a) Problem formulation; (b) adapted finite element mesh $($ nodes $=4843$, elements $=9441)$.

which is achieved depends upon the selection of the shock capturing viscosity (first derivative, second derivative of pressure etc.).

Figure 2 shows the Mach and pressure contours and the comparison of coefficient of pressure along the bottom line of the domain. The results predicted by the first gradient of pressure are slightly diffusive compared with the other methods. For the same grid used in the study, the anisotropic shock capturing (IV) and methods I and III give a good result with less diffusive effects.

In Figure 3, the approximate locations where the diffusion is added are depicted by plotting the contours of different coefficients used in the shock capturing techniques. In the first three shock capturing viscosities, the additional diffusion is based on the pressure distribution in the domain. In method I, the switch based on the nodal pressure values (Equation (16)) is plotted. In methods II and III, it is the scaled values of first and second gradients of pressure, respectively. The spatial residuals are plotted for method IV. It is seen that method I based on the pressure switch adds additional diffusion in the uniform region also. However, the results predicted by this method are good in most of the inviscid problems including the present problem. It is observed that all methods considered are good in locating the shock.

\subsection{Supersonic inviscid flow past a wedge}

The second example considered is the supersonic inviscid flow past a $15^{\circ}$ ramp as shown in Figure 4. A uniform mesh (Figure 4(a)) with 1780 nodes and 3396 elements and an adapted 
mesh with 168 nodes and 286 elements are used to solve the problem. The inlet Mach number has been taken as 3.0. The density, pressure and Mach contours predicted by the three artificial viscosity schemes have also been shown in the figure (Figure 4(d,e,f)). The results obtained from method II have not been provided, as it has shown diffuse solution, compared with all other methods. The other three methods predict the shock equally well.

Figure 5 shows the comparison of density, pressure and Mach number values (uniform mesh) along section AA for all schemes and analytical solution. Again all the three methods predict the results with almost same accuracy.

The solution obtained from the adapted mesh is shown in Figures 6 and 7. Here, even though the contours predicted by method II are slightly diffusive (Figure 6), the quantitative results (Figure 7) match well with the other results considered. It shows that the adaptive procedure improves the solution with any kind of shock capturing viscosity.

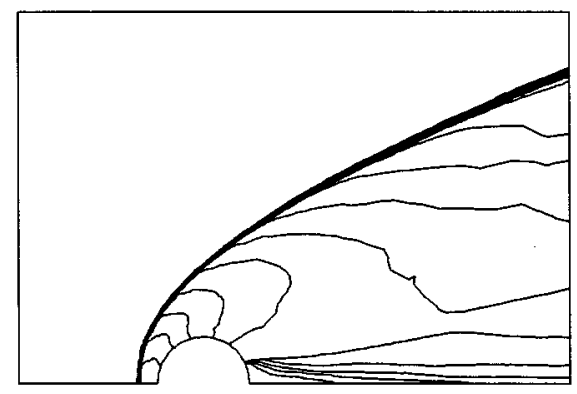

Method I

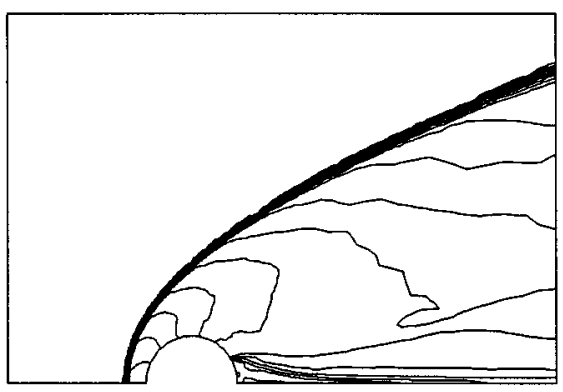

Method III

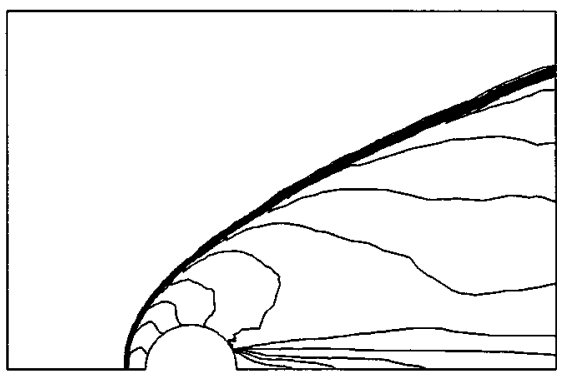

Method IV

Figure 16. Mach number contours for hypersonic flow past cylinder. 

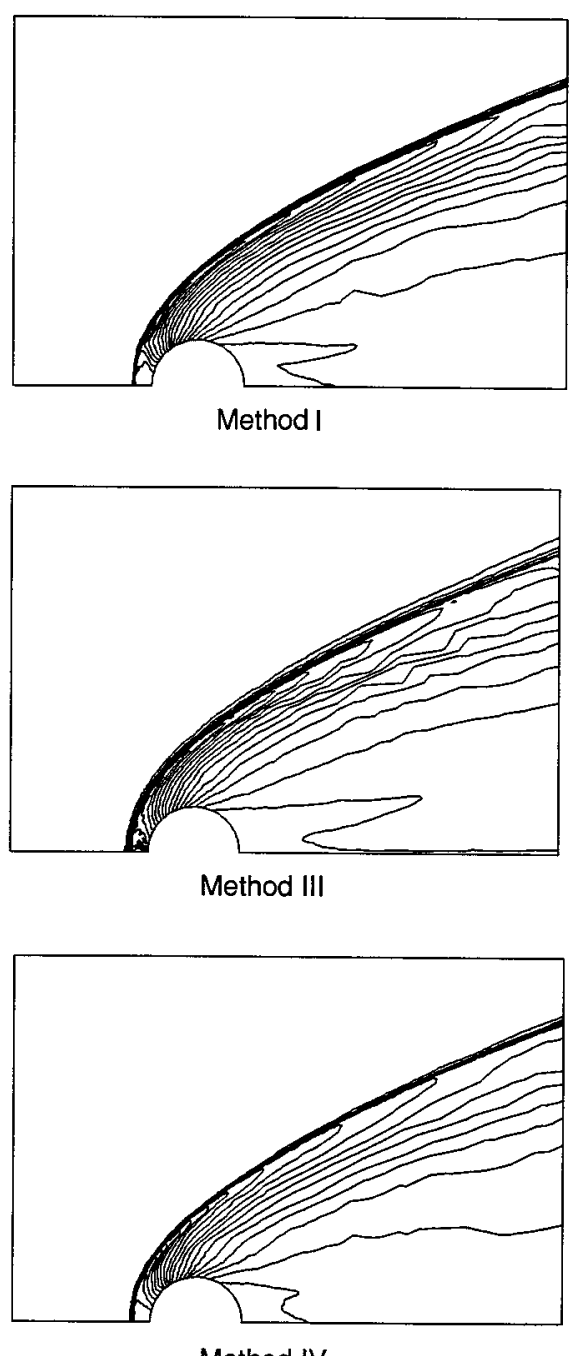

Method IV

Figure 17. Density contours for hypersonic flow past cylinder.

\subsection{Supersonic flow past NACA0012 aerofoil}

The problem formulation and the mesh generated are shown in Figure 8. A finite element mesh with more elements near the surface of the aerofoil has been generated. There are 3753 nodes and 7351 elements in the mesh (Figure 8(b)). The detail of the mesh near the surface is also shown (Figure 8(c)). The inlet Mach number is assumed to be 1.2. This Mach number is the smallest value ever considered in this study.

The results obtained for this problem are shown in Figures 9 and 10. All four methods considered give an equally good performance. This can be attributed to the low Mach number flow considered in the problem. 
5.4. Supersonic flow over a full cylinder at $M=3.0$

Figure 11 shows the problem formulation and adapted mesh used in the present computation. The figure also shows the Mach number contours obtained using methods I and IV. The inlet Mach number is assumed to be 3. The adapted mesh consists of 12651 nodes and 24979 elements. Such a fine mesh is essential for this problem to resolve the quasi-rarefaction zone. As this zone often creates a negative pressure zone, care must be taken to control the time step and additional shock capturing viscosity constants.

Both methods I and IV are seen to predict the shock almost in the same position. A difference has been observed in the pattern behind the cylinder. Even though there is no way to validate the results behind the cylinder, the prediction of method IV agrees well with the special schemes (see Reference [5]).

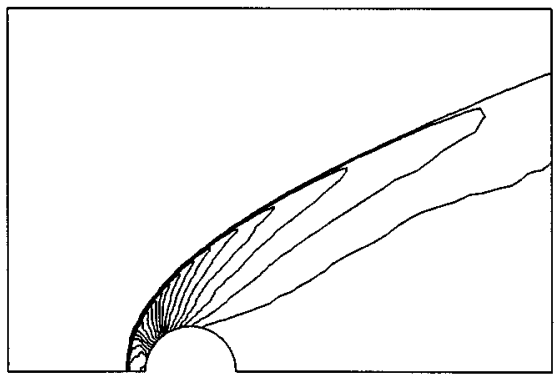

Method I

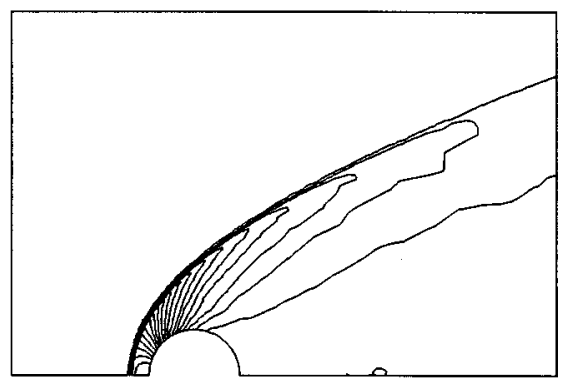

Method III

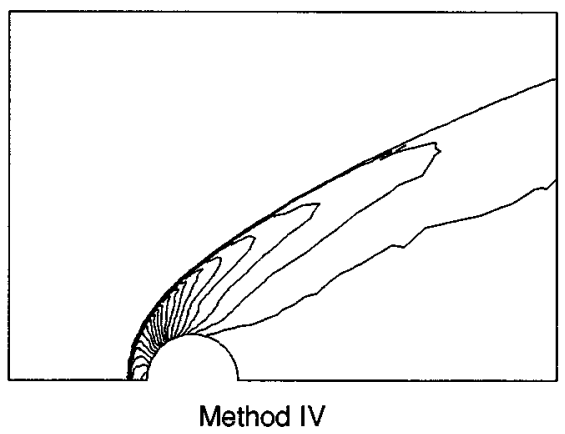

Figure 18. Pressure contours for hypersonic flow past cylinder. 


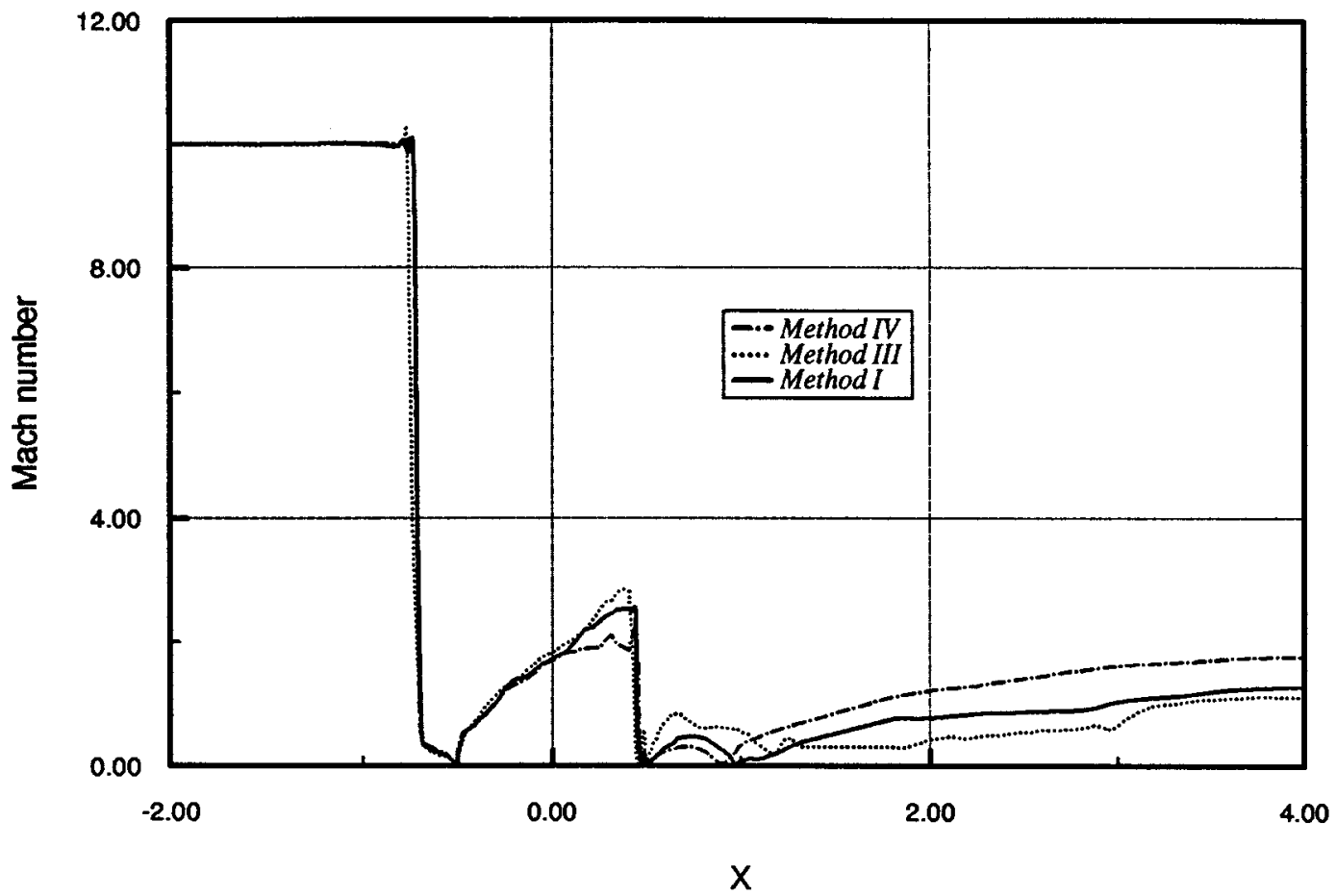

Figure 19. Mach number distribution along the bottom line for hypersonic flow past cylinder.

The quantitative results of the schemes are compared in Figure 12. The coefficient of pressure along the centerline of the domain and surface of the cylinder, calculated from both schemes, match excellently, except in the rear. Differences in prediction of the Mach number along the surface are observed between the two schemes. Method IV underpredicts the peak Mach number on the surface of the cylinder compared with method III and MUSCL scheme [5]. In this problem, direct use of anisotropic shock capturing (method IV) alone failed to give the Mach number peak on the cylinder surface. Small amount of residual smoothing along with the anisotropic shock capturing yields the present results comparable with other predictions.

\subsection{Hypersonic flow past a cylinder}

Another problem considered in the present study is the hypersonic inviscid flow past a quarter cylinder as shown in Figure 13. The inlet Mach number is assumed to be 6. The uniform mesh with 785 nodes and 1455 elements (Figure 13(b)) and an adapted mesh with 1391 nodes and 2637 elements (Figure 13(c)) are used to solve the problem.

Figure 13(d,e) show the Mach and pressure contours respectively, predicted by methods I and III using the uniform mesh. The anisotropic shock capturing (method IV), has not given a good solution with the uniform grid. Among the two methods presented, method I seems to give a better representation of shock. In general, the additional diffusion requirement of an inviscid problem is higher than that of a viscous problem. Here, the higher additional diffusion generated by method I gives a better answer than method III.

The results obtained from the adapted mesh are presented in Figure 14. The anisotropic shock capturing gives a reasonable solution. For the sake of comparison, the Mach number 
distribution from both initial and final meshes of Reference [21] is plotted along with the present predictions in Figure 14(c). With a relatively lower mesh density, present predictions compare well with the final adapted mesh of Reference [21]. Both the methods based on the second gradient of pressure (methods I and III) seem to give better results compared with the anisotropic shock capturing (method IV).

\subsection{Hypersonic flow past a half cylinder}

Hypersonic flow past a half cylinder has been considered as the next example in the present computation. The problem formulation, finite element mesh and the contour plots are shown in Figures 15-18. The inlet Mach number is assumed to be 10 and the fluid is inviscid. An adapted mesh with 4843 nodes and 9441 elements is used to solve the problem.

All three methods considered predict the shock in the same position in front of the cylinder (Figure 16). The resolution of the shock predicted by method I is better than any other scheme. This quality is probably due to the pressure switch used in this method. The density and pressure contours are shown in Figures 17 and 18 respectively. From these contour plots, method IV gives a better performance than method III.

The Mach number distribution along the bottom line of the domain is shown in Figure 19. A small oscillation in the vicinity of the shock is observed when method III is used. Also, this

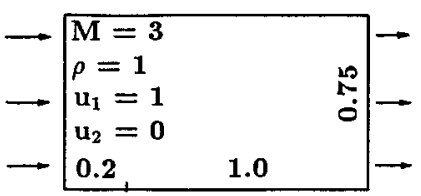

(a)
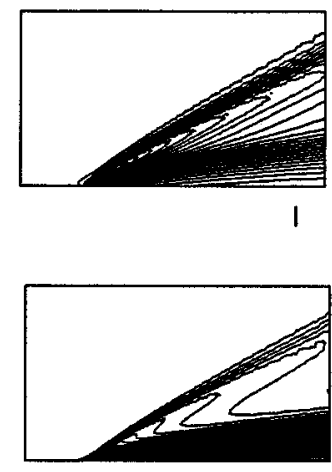

1

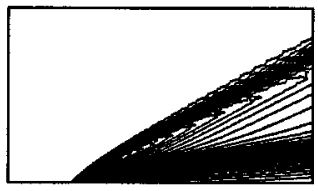

(c)

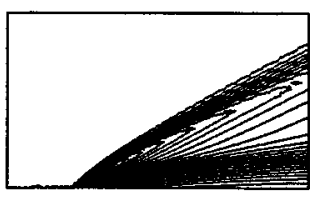

II

(c)

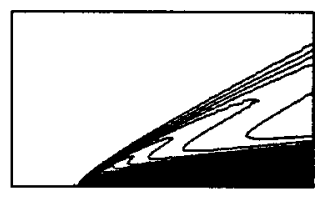

II

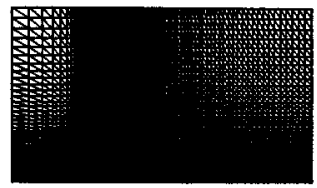

(b)

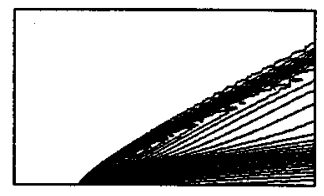

III

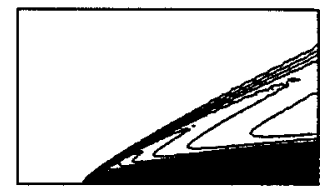

III

(d)

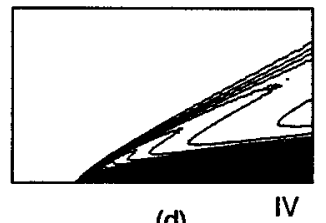

(d)

Figure 20. Supersonic viscous flow past a flat plate $(M=3, R e=1000)$. (a) Problem formulation; (b) structured finite element mesh (nodes $=6750$, elements $=13172$ ); (c) density contours; and (d) Mach number contours. 


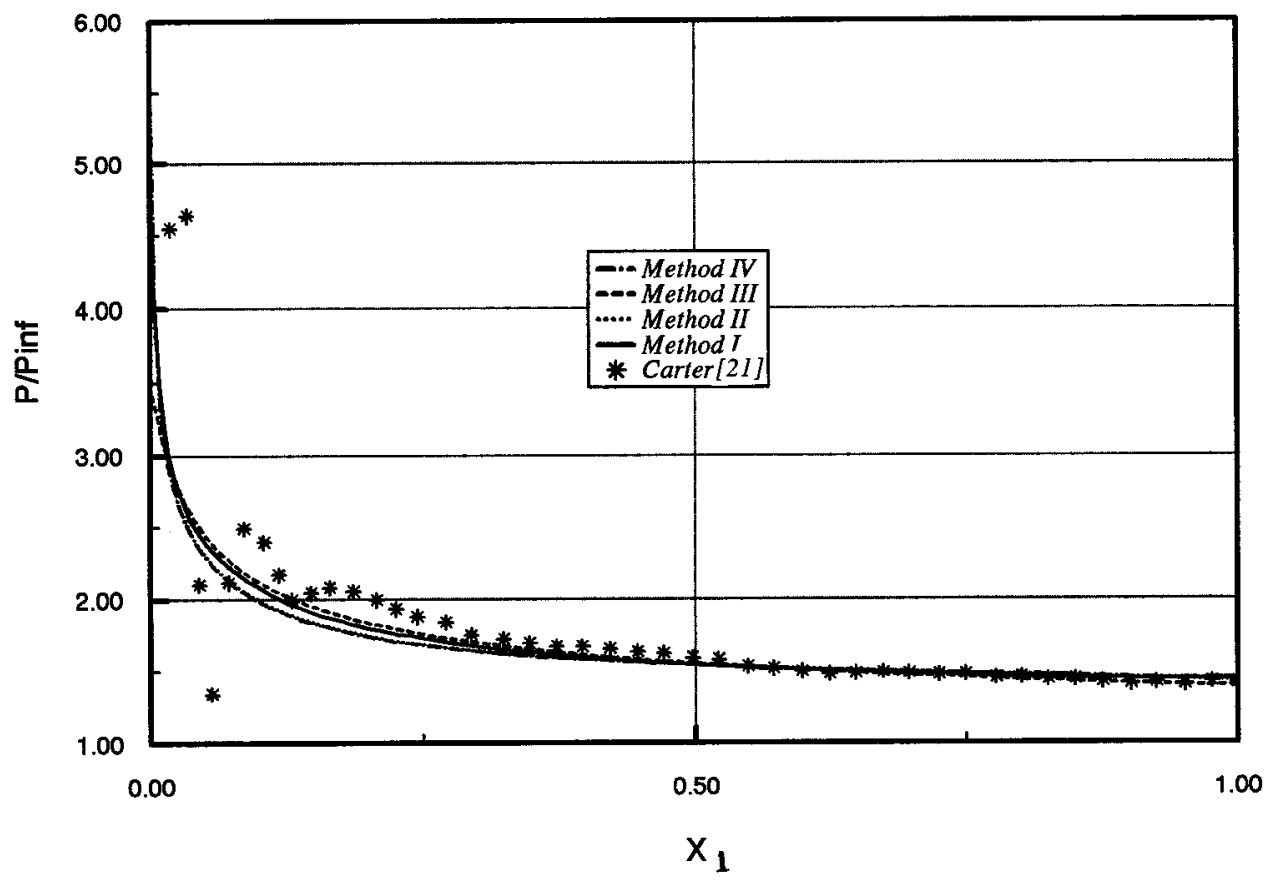

(a)

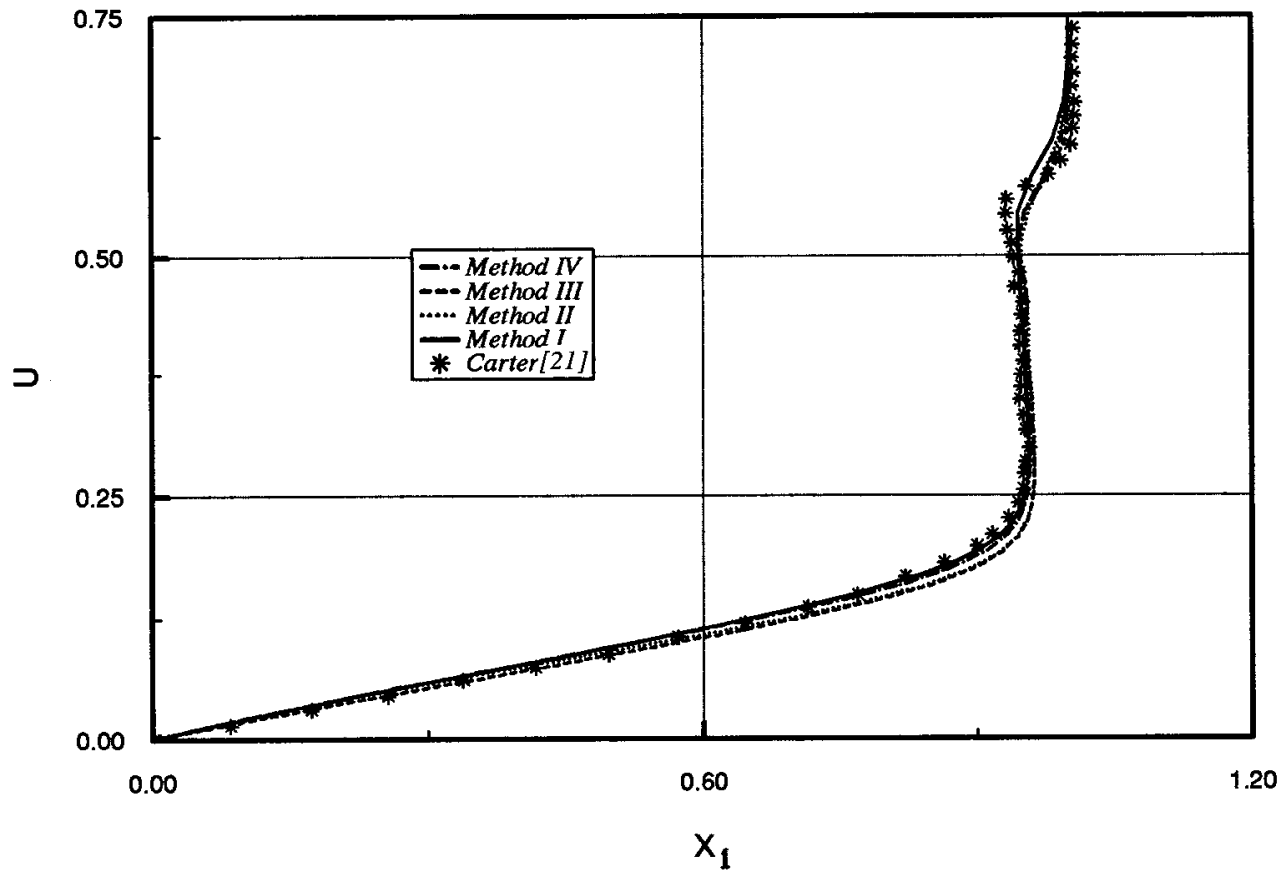

(b)

Figure 21. Comparison of present predictions with Carter [21]. (a) Pressure distribution along the surface; (b) outlet velocity profile along the surface. 

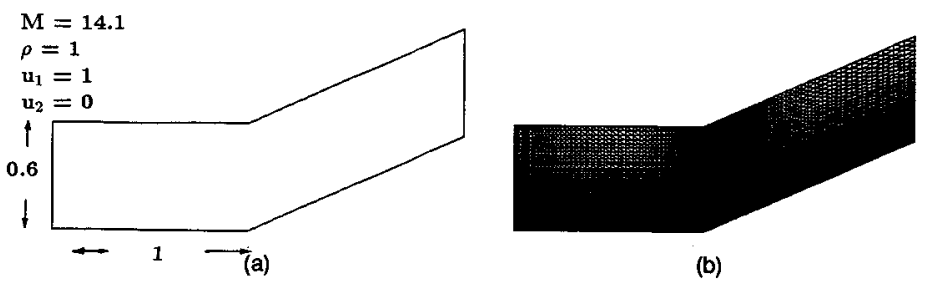

(b)
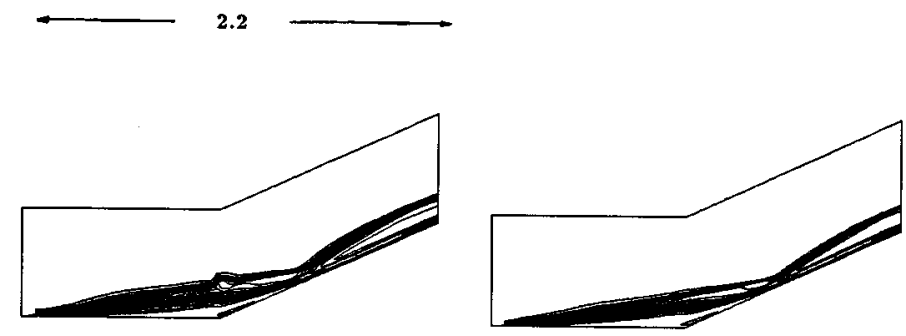

(c)

III
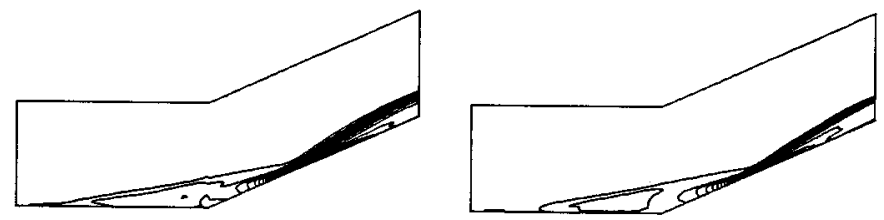

(d)

III

Figure 22. Hypersonic viscous flow past a $24^{\circ}$ compression corner $(M=14.1, R e=103680)$. (a) Problem formulation; (b) finite element mesh (nodes =11211, elements =22000; (c) Mach number contours; and (d) pressure contours.

method fails to give a clear recirculation behind the cylinder. All three methods differ in predicting the Mach number along the surface of the cylinder and behind it. From the patterns observed, methods I and IV are seen to give a better recirculation with three stagnation points behind the cylinder.

\subsection{Supersonic laminar viscous flow past a flat plate}

Figure 20 shows problem formulation and the results obtained for the supersonic viscous flow past a flat plate. Here, the Reynolds number based on the length of the plate is 1000 and the free stream Mach number is assumed to be 3. The Prandtl number is taken as 0.72 . The temperature of the plate is assumed constant and equal to the stagnation temperature and is given as

$$
T_{\mathrm{w}}=\frac{1}{(\gamma-1) M_{\infty}^{2}}\left(1+\frac{\gamma-1}{2} M_{\infty}^{2}\right) .
$$

A structured mesh with higher density near the plate surface has been generated (Figure 20(b)). The first node from the surface of the plate is placed at a distance of 0.0004 from the surface. In total, there are 6750 nodes and 13172 elements in the mesh.

The density and Mach contours obtained from all shock capturing techniques are shown in Figure 20(c,d). All of the schemes give an equally good performance in this problem. However, method II gives a slightly diffuse solution compared with all other schemes. 


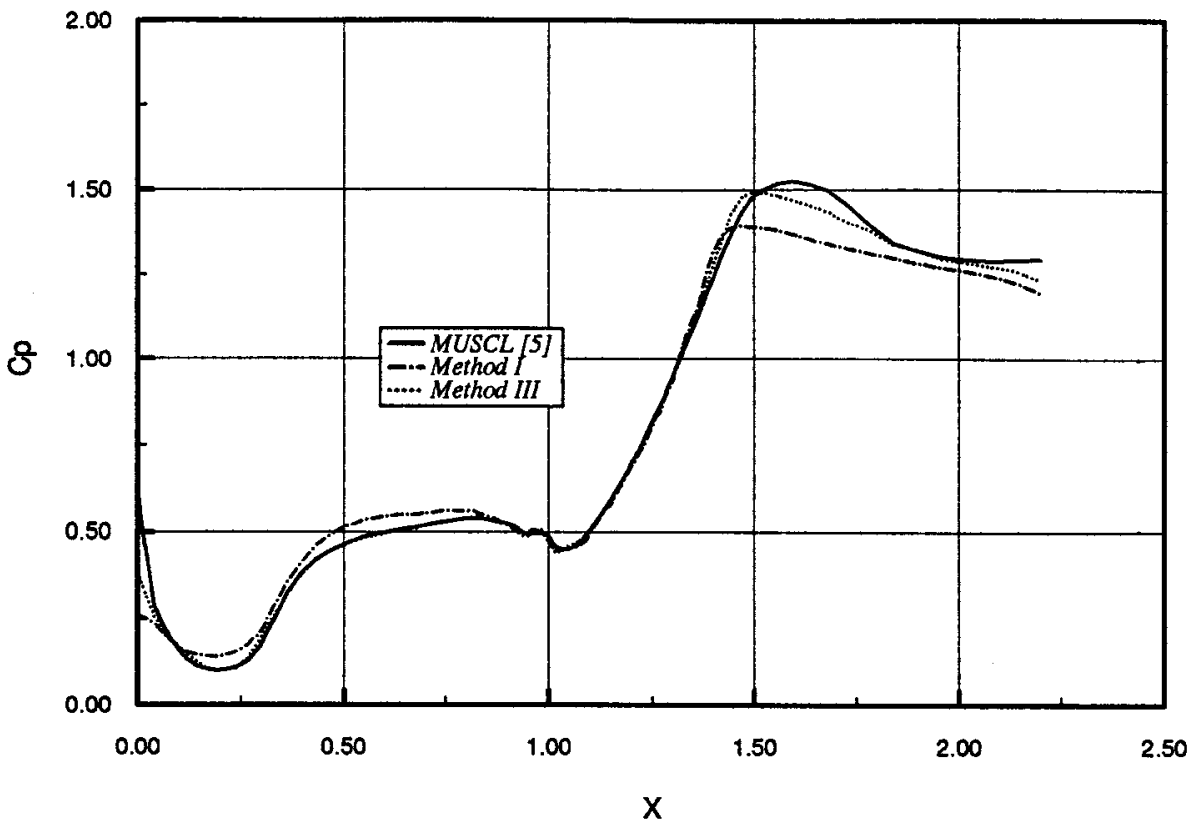

(a)

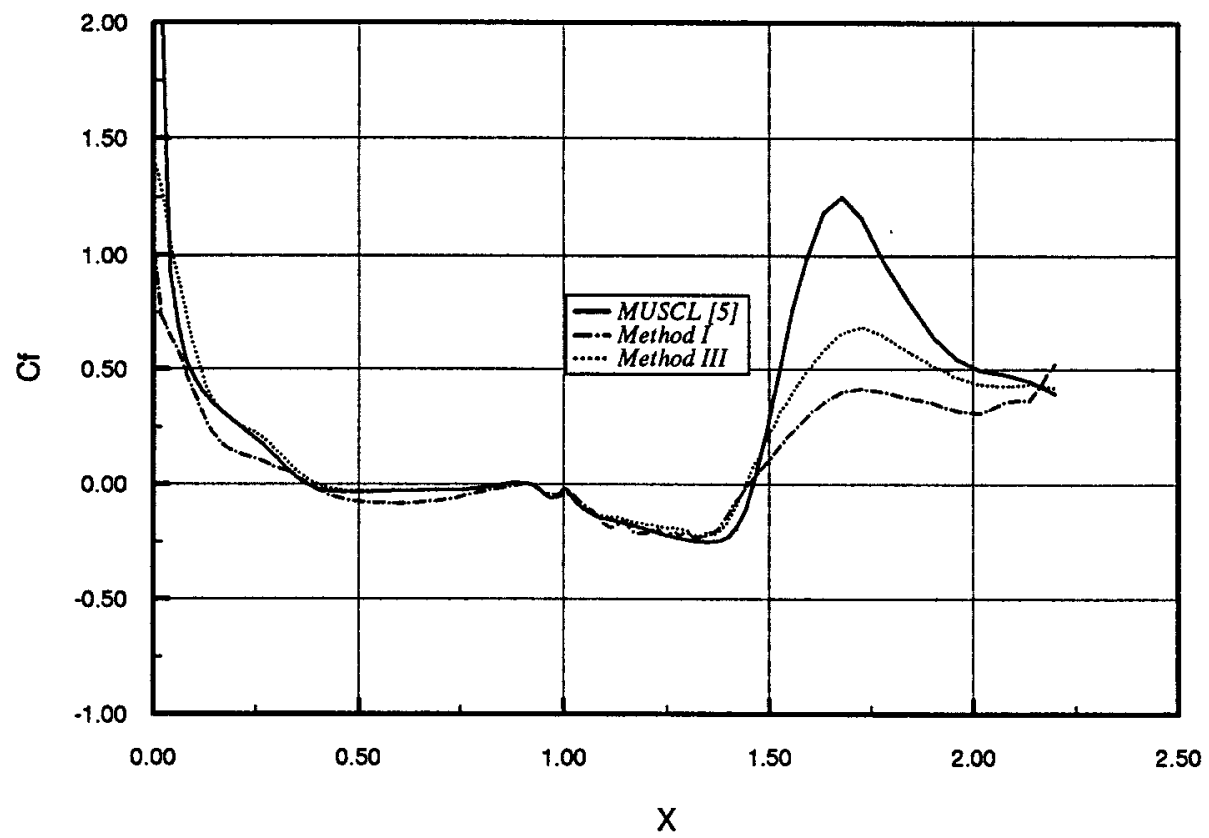

(b)

Figure 23. Hypersonic flow past a compression corner. (a) Comparison of coefficient of pressure along the wall; (b) comparison of skin-friction coefficient along the wall. 
Figure 18 shows the quantitative comparison obtained for all of the shock capturing techniques with those of Carter [21]. Present predictions (Figure 21(a)) of non-dimensional pressure along the surface of the plate is seen to agree well with Carter [21] towards the trailing edge of the plate. Differences do exist between the present predictions and those of Carter near leading edge. However, no oscillations are observed in the present predictions. The outlet velocity profile (Figure 21(b)) is also seen to compare excellently. It is seen that method III gives a better comparison of pressure distribution with Carter [21].

\subsection{Hypersonic viscous flow past a compression corner}

The final test case considered in the present study is the hypersonic viscous flow past a compression corner of $24^{\circ}$. The free stream is at a Mach number of 14.1 and the Reynolds number is 103680 , based upon the horizontal flat plate length of $1.44 \mathrm{ft}$. The temperature of the free stream and wall are fixed at 160 and $535^{\circ}$ Rankine respectively. The fluid Prandtl number is constant and equal to 0.72 . Figure 22 (a) shows the details of formulation. In Figure 22(b), the mesh generated is shown. This is a structured mesh with $111 \times 101$ nodes.

Figure 22(c,d) shows the Mach and pressure contours respectively. The contours predicted by method I are generally diffusive than those predicted by method III. It shows that method III gives a better performance than method I in viscous computations. This can be attributed to the controlled diffusion added by method III rather than that of method I.

The quantitative results predicted are shown in Figure 23(a,b). The coefficients are calculated using the relations

$$
\begin{aligned}
& C_{p}=\log \left[\frac{50 p_{\mathrm{w}}}{\rho_{\infty} u_{\infty}^{2} / 2}\right], \\
& C_{f}=50\left[\frac{\tau_{\mathrm{w}}}{\rho_{\text {infty }} u_{\infty}^{2} / 2}\right],
\end{aligned}
$$

where $\mathrm{w}$ and $\infty$ refer to the wall and free stream, respectively.

$$
\tau_{\mathrm{w}}=t_{2}^{(n)} n_{1}-t_{1}^{(n)} n_{2}=\left(\tau_{21} n_{1}+\tau_{22} n_{2}\right) n_{1}-\left(\tau_{11} n_{1}+\tau_{12} n_{2}\right) n_{2}
$$

It is seen from the comparison that the patterns of variation observed for both of the shock capturing viscosities considered are similar. However, method III shows a better comparison with MUSCL scheme [5] than method I (Figure 23).

\section{CONCLUSIONS}

Among the first three families of shock capturing viscosities, the procedures based on the second gradient of pressure are best schemes and are suitable for almost all type of applications. Method $\mathrm{I}$ is an excellent choice for compressible inviscid flow problems. However, this method's performance in high speed viscous problems is not very good. From the study carried out, the method based on $L_{2}$ projection (second gradient of pressure, method III) has given the best performance for viscous high speed compressible flows. The anisotropic shock capturing (method IV) gave an excellent performance in the supersonic range. However, at high Mach numbers, this method needs small amount of residual smoothing to get acceptable answers. Method II (first gradient of pressure) has also given good results at low Mach number flows. But its performance is not so attractive compared with other methods considered in the present study. 


\section{REFERENCES}

1. C. Hirsch, Numerical Computation of Internal and External Flows, Vol. 2, Wiley, New York, 1990.

2. P. Woodward and P. Colella, 'The numerical simulation of two-dimensional fluid flow with strong shocks', $J$. Comput. Phys., 54, 115-173 (1984).

3. P.R.M. Lyra, K. Morgan, J. Peraire and J. Peiro, 'TVD algorithms for the solution of the compressible Euler equations on unstructured meshes', Int. J. Numer. Methods Fluids, 19, 827-847 (1994).

4. M.T. Manzari, 'An unstructured grid finite element algorithm for compressible turbulent flow computations', Ph.D Thesis, University of Wales, Swansea, 1996.

5. B.V.K. Satya Sai, O.C. Zienkiewicz, M.T. Manzari, P.R.M. Lyra and K. Morgan, 'General purpose vs special algorithms for high speed flows with shocks', Report CR/935t96, Inst. Numer. Methods Eng., University of Swansea, April 1996.

6. J. Von Neumann and R.D. Richtmyer, 'A method for the numerical calculations of hydrodynamical shocks', $J$. Math. Phys., 21, 232-237 (1950).

7. A. Lapidus, 'A detached shock calculation by second-order finite differences', J. Comput. Phys., 2, 154-177 (1967).

8. J.L. Steger, 'Implicit finite difference simulation if flow about two-dimensional geometries', AIAA J., 16, 679-686.

9. R.W. MacCormack and B.S. Baldwin, 'A numerical method for solving the Navier-Stokes equations with application to shock-boundary layer interaction', AIAA Paper 75-1, 1975.

10. A. Jameson and W. Schmidt, 'Some recent developments in numerical methods in transonic flows', Comput. Mesh. Appl. Mech. Eng., 51, 467-493 (1985).

11. J. Peraire, J. Peiro, L. Formaggia, K. Morgan and O.C. Zienkiewicz, 'Finite element Euler computations in three dimensions', Int. J. Numer. Methods Eng., 26, 2135-2159 (1988).

12. K. Morgan, J. Peraire, J. Peiro and O.C. Zienkiewicz, 'Adaptive remeshing applied to the solution of a shock interaction problem on a cylindrical leading edge', in P. Stow (ed.), Computational Methods in Aeronautical Fluid Dynamics, Clarenden Press, Oxford, 1990, pp. 327-344.

13. O.C. Zienkiewicz, B.V.K. Satya Sai, K. Morgan, R. Codina and M. Vázquez, 'A general algorithm for compressible and incompressible flow-Part II. Tests on the explicit form', Int. J. Numer. Methods Fluids, 20, $887-913$ (1995).

14. O.C. Zienkiewicz and J. Wu, 'A general explicit of semi-explicit algorithm for compressible and incompressible flows', Int. J. Numer. Methods Eng., 35, 457-479 (1992).

15. R. Codina, 'A discontinuity-capturing crosswind-dissipation for the finite element solution of the convection diffusion equation', Comput. Methods Appl. Mech. Eng., 110, 325-342 (1993).

16. O.C. Zienkiewicz and R. Codina, 'A general algorithm for compressible and incompressible flow, Part I. The split characteristic based scheme', Int. J. Numer. Methods Fluids, 20, 869-885 (1995).

17. O.C. Zienkiewicz, B.V.K. Satya Sai, K. Morgan and R. Codina, 'Split characteristic based semi-implicit algorithm for laminar/turbulent incompressible flows', Int. J. Numer. Methods Fluids, 23, 1-23 (1996).

18. R. Codina, M. Vásquez and O.C. Zienkiewicz, 'General algorithm for compressible and incompressible flows, Part III-A semi-implicit form', to be published in Int. J. Numer. Methods Fluids.

19. O.C. Zienkiewicz and R.L. Taylor, The Finite Element Method, Vol. 2, 4th edn, McGraw-Hill, New York, 1991.

20. R. Lohner, K. Morgan and O.C. Zienkiewicz, 'The solution of non-linear hyperbolic equation systems by the finite element method', Int. J. Numer. Methods Fluids, 4, 1043-1063 (1984).

21. J.E. Carter, 'Numerical solutions of the Navier-Stokes equations for the supersonic laminar flow over a two-dimensional compression corner', NASA TR-R-385, 1972. 